\title{
COMPACTIFICATIONS OF SPACES OF FUNCTIONS AND INTEGRATION OF FUNCTIONALS
}

BY

\section{S. GRINBLAT}

ABSTRACT. For a locally compact space there exists a compactification such that all its points are effectively describable, namely, Alexandroff's onepoint compactification. The effective construction of compactifications for numerous standard separable metric spaces is already a very nontrivial problem. We propose a method of compactification which enables us to effectively construct compactifications of some spaces of functions (for example, of a ball in $\left.L_{p}(-\infty, \infty)\right)$. It will be shown that the study of compactifications of spaces of functions is of principal importance in the theory of integration of functionals and in limit theorems for random processes.

0. Introduction. The space $X$ is the space of all Lebesgue measurable functions defined on the interval $[0,1]$ and such that $0 \leqslant x(t) \leqslant 1$; we denote them by $x(t)$ or simply $x$. The metric on $X$ is determined by

$$
\rho\left(x_{1}, x_{2}\right)=\left(\int_{0}^{1}\left[x_{1}(t)-x_{2}(t)\right]^{2} d t\right)^{1 / 2}
$$

DEFINITION 0.1. The functional

$$
F(x)=\int_{0}^{1} \cdots \int_{0}^{1} \phi\left(t_{1}, \ldots, t_{k} ; x\left(t_{1}\right), \ldots, x\left(t_{k}\right)\right) d t_{1} \cdots d t_{k}
$$

is called a functional of the integral form, where $\phi\left(t_{1}, \ldots, t_{k} ; x_{1}, \ldots, x_{k}\right)$ is a continuous function on the unit cube of dimension $2 k\left(0 \leqslant t_{1} \leqslant 1, \ldots, 0 \leqslant\right.$ $\left.t_{k} \leqslant 1 ; 0 \leqslant x_{1} \leqslant 1, \ldots, 0 \leqslant x_{k} \leqslant 1\right)$ and $k$ is any positive integer.

The following is one of the main results of this paper.

There exists a metric compactification $g X$ of the space $X$ such that any bounded continuous functional which can be continuously extended on $g X$ is the uniform limit of the functionals of the integral form. Any functional of integral form can be continuously extended on $g X$. Every point in $g X$ can be written as $a_{\psi}$, where $\psi(t, x)$ is a Lebesgue measurable function $(0 \leqslant t \leqslant 1,0 \leqslant$ $x \leqslant 1,0 \leqslant \psi(t, x) \leqslant 1$ ) such that for the functional of the integral form $F(x)$ we have

Received by the editors December 9, 1974.

AMS (MOS) subject classifications (1970). Primary 46J10, 54D35, 28A40, 60B10.

Copyright $\odot$ 1976, American Mathematical Society 


$$
F\left(a_{\psi}\right)=\int_{0}^{1} \cdots \int_{0}^{1} \phi\left(t_{1}, \ldots, t_{k} ; \psi\left(t_{1}, x_{1}\right), \ldots, \psi\left(t_{k}, x_{k}\right)\right) d t_{1} \cdots d t_{k} d x_{1} \cdots d x_{k} .
$$

This result is an example of use of the method of compactification, which is described in $\S 1$.

The integration of the functionals is studied in $\$ 2$. For the functional $F(x)$ of the integral form we have

$$
T F=\int_{0}^{1} \cdots \int_{0}^{1} \phi\left(t_{1}, \ldots, t_{k} ; x_{1}, \ldots, x_{k}\right) d t_{1} \cdots d t_{k} d x_{1} \cdots d x_{k}=F\left(a_{x}\right),
$$

where $T F$ is the Gâteaux-Levy integral of the functional $F(x)$ and the point $a_{x} \in g X$.

Various examples of integrable and nonintegrable functionals are investigated in $\$ 3$.

The exact knowledge of the compactification $g X$ enables us to make a few assertions on various compactifications of the space $X$. This is done in $\S 4$.

The limit theorems for the measurable random processes are studied in $\S 5$. Of special interest is the following result of $\S 5$. Let $\xi_{1}(t), \ldots, \xi_{n}(t), \ldots$ and $\xi(t)$ be measurable random processes $\left(0 \leqslant t \leqslant 1,0 \leqslant \xi_{n}(t) \leqslant 1,0 \leqslant \xi(t) \leqslant 1\right)$. Let $F_{m}$ be the sequence of the functionals of integral form. For any function $x_{0}(t) \in X$ and for any Lebesgue measurable function $\psi(t, x)(0 \leqslant t \leqslant 1,0 \leqslant x$ $\leqslant 1,0 \leqslant \psi(t, x) \leqslant 1)$, where $x_{0}(t) \neq \psi(t, x)$, there exists a number $m^{\prime}$ such that $F_{m^{\prime}}\left(x_{0}(t)\right) \neq F_{m^{\prime}}\left(a_{\psi}\right)$. The theorem states, that if for any $m$ the joint distributions of the random variables $F_{1}\left(\xi_{n}(t)\right), \ldots, F_{m}\left(\xi_{n}(t)\right)$ converge weakly to the joint distribution of the random variables $F_{1}(\xi(t)), \ldots, F_{m}(\xi(t))$, then for any bounded continuous functional $F(x)$ we have

$$
\lim _{n \rightarrow \infty} E F\left(\xi_{n}(t)\right)=E F(\xi(t)),
$$

where $E$ denotes, as usual, the mathematical expectation. The proof is based on the fact that the closure of the algebra, generated by the functionals $F_{m}(x)$, is the Banach algebra of bounded continuous functionals, which defines the compactification of $\left.X .{ }^{1}\right)$

The compactifications for some spaces of functions are studied in $\$ 6$.

In conclusion, I want to express my gratitude to Professor H. Furstenberg for his invaluable assistance.

1. Functionals of integral forms and a method of compactification. Continuity of functionals on $X$ is considered with respect to the metric (0.1). In the introduction we have already considered functionals of integral form. The functionals of integral form are obviously continuous and bounded on $X$. If $F(x)$ is

(1) Throughout this paper, an algebra will mean an algebra with identity. "The algebra $A$ is generated by the set $\left\{a_{\lambda}\right\}$ ", will mean, " $A$ is generated by the set $\left\{a_{\lambda}\right\}$ and the identity". 
such a functional, then of course $d \cdot F(x)$ ( $d$-real number) is also a functional of integral form. Let two functionals of integral form,

$$
\begin{aligned}
& F_{1}(x)=\int_{0}^{1} \cdots \int_{0}^{1} \phi_{1}\left(t_{1}, \ldots, t_{k_{1}} ; x\left(t_{1}\right), \ldots, x\left(t_{k_{1}}\right)\right) d t_{1} \cdots d t_{k_{1}}, \\
& F_{2}(x)=\int_{0}^{1} \cdots \int_{0}^{1} \phi_{2}\left(t_{1}, \ldots, t_{k_{2}} ; x\left(t_{1}\right), \ldots, x\left(t_{k_{2}}\right)\right) d t_{1} \cdots d t_{k_{2}},
\end{aligned}
$$

be given. Set $k_{1} \geqslant k_{2}$. Then

$$
\begin{aligned}
& F_{1}(x)+F_{2}(x) \\
& =\int_{0}^{1} \cdots \int_{0}^{1}\left[\phi_{1}\left(t_{1}, \ldots, t_{k_{1}} ; x\left(t_{1}\right), \ldots, x\left(t_{k_{1}}\right)\right)\right. \\
& \left.+\phi_{2}\left(t_{1}, \ldots, t_{k_{2}} ; x\left(t_{1}\right), \ldots, x\left(t_{k_{2}}\right)\right)\right] d t_{1} \cdots d t_{k_{1}}, \\
& F_{1}(x) \cdot F_{2}(x) \\
& =\int_{0}^{1} \cdots \int_{0}^{1}\left[\phi_{1}\left(t_{1}, \ldots, t_{k_{1}} ; x\left(t_{1}\right), \ldots, x\left(t_{k_{1}}\right)\right)\right. \\
& \left.\times \phi_{2}\left(t_{k_{1}+1}, \ldots, t_{k_{1}+k_{2}} ; x\left(t_{k_{1}+1}\right), \ldots, x\left(t_{k_{1}+k_{2}}\right)\right)\right] \\
& d t_{1} \cdots d t_{k_{1}+k_{2}} \text {. }
\end{aligned}
$$

Thus the sum and the product of two functionals of integral form are also functionals of integral form. Hence we get an algebra over the field of real numbers. The uniform closure of this algebra is a certain Banach algebra of bounded continuous functionals, which we denote by $G(X)$.

Let us review some facts of general topology. Let $\hat{X}$ be a completely regular topological space. The compact set $K$ will be said to be a compactification of $\hat{X}$ if there exists a homeomorphism from $\hat{X}$ to a dense subset in $K$. Let $C(K)$ be the Banach algebra of all bounded continuous functions $K$. Obviously $C(K)$ is a Banach algebra of bounded continuous functions on $\hat{X}$. We say that $C(K)$ determines the compactification of $\hat{X}$.

TyChonoff's TheOREM (SEE [9, VII, §43]). The Banach algebra $A(\hat{X})$ of bounded continuous functions on a completely regular topological space $\hat{X}$ determines a compactification of this space if and only if for any point $x_{0} \in \hat{X}$ and any closed set $Y \subset \hat{X}, x_{0} \notin Y$, there exists a function $f \in A(\hat{X})$ such that $f\left(x_{0}\right)=0$ and $f(x)=1$ for all $x \in Y$.

Since $X$ is metric it is completely regular.

THEOREM 1.1. The algebra $G(X)$ determines a compactification of $X$ which will be a metric space.

Proof. Let $x_{0} \in X$. Consider the functional 


$$
F_{0}(x)=\int_{0}^{1}\left[x_{0}(t)-x(t)\right]^{2} d t
$$

Let $x_{n}(t)$ be a sequence of continuous functions belonging to $X$ such that $x_{n}(t)$ converges almost everywhere to $x_{0}(t)$. It is evident that the functionals

$$
F_{n}(x)=\int_{0}^{1}\left[x_{n}(t)-x(t)\right]^{2} d t
$$

are functionals of integral form. The functionals $F_{n}(x)$ converge uniformly to the functional $F_{0}(x)$ and so $F_{0}(x) \in G(X)$. If $Y$ is a closed set in $X$ and $x_{0} \notin Y$, then $\rho\left(x_{0}, Y\right)=\alpha>0$. The functional

$$
F_{0}^{\prime}(x)=\alpha^{-2} \min \left(\alpha^{2}, F_{0}(x)\right)
$$

belongs to $G(X) ; F_{0}^{\prime}\left(x_{0}\right)=0 ; F_{0}^{\prime}(x)=1$ if $x \in Y$.

Thus, by virtue of Tychonoff's Theorem, $G(X)$ determines a compactification of $X$, which we denote by $g X$. Since $G(X)$ is separable, it follows from Urysohn's theorem (see $[4$, p. 388]) that $g X$ is a compact metric space. The countable dense set in $G(X)$ is given, for example, by all the functionals of the form (0.2), where $\phi\left(t_{1}, \ldots, t_{k} ; x_{1}, \ldots, x_{k}\right)$ is a polynomial with rational coefficients. Q.E.D.

Any continuous function on $g X$ restricted to $X$ is a functional in $G(X)$. Conversely, any functional in $G(X)$ has a unique continuous extension to $g X$. Hence we can speak about the value of a functional from $G(X)$ in any point of $g X$. Any point in $g X$ determines a maximal ideal in $G(X)$, i.e. the set of all functionals from $G(X)$ which vanish at this point. For any maximal ideal in $G(X)$ there exists a point in $g X$ which determines this ideal. Any point $x$ in $g X$ determines a linear multiplicative continuous functional $f$ on $G(X)$, whose value on the functional 1 is equal to 1 , i.e. $f(F)=F(x)$ for any $F \in G(X)$. Any linear multiplicative continuous functional on $G(X)$, its value on the functional 1 being equal to 1 , is determined by some point in $g X$.

Now we pass to the main construction of the present paper. Let $Z$ be the unit square $(0 \leqslant t \leqslant 1,0 \leqslant z \leqslant 1)$. Let $M$ be the set of all finite countably additive measures $\mu$ defined on the Borel sets of the unit square $Z$ such that $\mu(Z)$ $=1$. As usual we shall say that the sequence of measures $\mu_{1}, \ldots, \mu_{n}, \ldots$ belonging to $M$ converges weakly to the measure $\mu \in M$ if for any function $f(t, z)$ continuous on $Z$ we have

$$
\lim _{n \rightarrow \infty} \int f(t, z) d \mu_{n}=\int f(t, z) d \mu .
$$

The convergence (1.5) makes $M$ a compact metric space.

Let $x \in X$. Consider the following measure $\mu_{x} \in M$. If $A$ is a Borel set in $Z$, then

$$
\mu_{x}(A)=\int_{0}^{1} \chi_{A}(t, x(t)) d t
$$


where $\chi_{A}(t, z)$ is a characteristic function of the set $A$, i.e. $\chi_{A}(t, z)=1$ if $(t, z) \in A$, and $\chi_{A}(t, z)=0$ for $(t, z) \notin A$. The closure in $M$ of the set of all measures $\mu_{x}$ is a compact metric space, which we denote by $M^{*}$. It can be easily checked that the mapping $x \rightarrow \mu_{x}$ is a homeomorphism of the space $X$ onto a dense subset of $M^{*}$. Thus $M^{*}$ is a compactification of $X$.

We assert that the compactifications of $g X$ and $M^{*}$ are equivalent, i.e. there exists a homeomorphism of $g X$ onto $M^{*}$ such that to every function $x \in X \subset g X$ there corresponds the measure $\mu_{x}$.

Consider on $X$ the functionals of the integral form

$$
F_{n, m}(x)=\int_{0}^{1} t^{n} x^{m}(t) d t
$$

$(n=0,1,2, \ldots, m=0,1,2, \ldots)$. Functionals (1.6) are the generating elements of an algebra of functionals, its uniform closure being $G(X)$, since this algebra contains all the functionals of integral form $(0.2)$ such that the function $\phi\left(t_{1}, \ldots, t_{k} ; x_{1}, \ldots, x_{k}\right)$ is a polynomial. It can be easily seen that

$$
F_{n, m}(x)=\int t^{n} z^{m} d \mu_{x}
$$

Hence the functional $F_{n, m}(x)$ can be continuously extended to the compact set $M^{*}$ if we set

$$
F_{n, m}\left(\mu^{*}\right)=\int t^{n} z^{m} d \mu^{*}
$$

for all $\mu^{*} \in M^{*}$. Consider two distinct measures $\mu_{1}^{*}, \mu_{2}^{*} \subset M^{*}$. There exists a functional of the form (1.6) such that

$$
F_{n, m}\left(\mu_{1}^{*}\right)=\int t^{n} z^{m} d \mu_{1}^{*} \neq \int t^{n} z^{m} d \mu_{2}^{*}=F_{n, m}\left(\mu_{2}^{*}\right) .
$$

According to the Stone theorem (see [8]) a Banach algebra $C^{\prime}(K)$ of continuous functions on the compact set $K$ is the Banach algebra $C(K)$ of all continuous functions on $K$ if for any two distinct points $k_{1}, k_{2} \subset K$ there exists a function $f \in C^{\prime}(K)$ such that $f\left(k_{1}\right) \neq f\left(k_{2}\right)$. In view of the previous arguments and Stone's theorem, it follows that the algebra of all bounded continuous functionals on $X$ which can be continuously extended on $M^{*}$ is $G(X)$. Now according to the Gel'fand-Kolmogoroff theorem $\left(^{2}\right.$ ) (see [5]) it follows that the compactifications $g X$ and $M^{*}$ are equivalent.

Let us investigate the compact set $M^{*}$. For any $x \in X$ and any $0 \leqslant \alpha_{1}<$ $\alpha_{2} \leqslant 1$ we have

$$
\mu_{x}\left\{(t, z): t \in\left[\alpha_{1}, \alpha_{2}\right]\right\}=\alpha_{2}-\alpha_{1} \text {. }
$$

$\left({ }^{2}\right)$ Gel'fand-Kolmogoroff's theorem asserts only the existence of the homeomorphism between $g X$ and $M^{*}$, but the method of the proof implies that in this homeomorphism the space $X$ is "fixed". 
Hence for any $\mu^{*} \in M^{*}$ we have

$$
\mu *\left\{(t, z): t \in\left[\alpha_{1}, \alpha_{2}\right]\right\}=\alpha_{2}-\alpha_{1} .
$$

Conversely, if $\mu^{*} \in M$ and $\mu^{*}$ satisfies (1.7) for any interval $\left[\alpha_{1}, \alpha_{2}\right] \subset[0,1]$, then there exists a sequence of measures $\mu_{x_{n}}$ converging weakly to $\mu^{*}$ and thus $\mu^{*} \in M^{*}$. To see this, let us fix the natural number $n$ and divide the square $Z$ into $n^{2}$ sets $Z_{i j}(1 \leqslant i \leqslant n, 1 \leqslant j \leqslant n)$, where

$$
Z_{i j}=\left\{(t, z): \frac{i-1}{n} \leqslant t<\frac{i}{n} ; \frac{j-1}{n} \leqslant z<\frac{j}{n}\right\}
$$

if $i \neq n$ and $j \neq n$, and if for example $i=n$, then $Z_{n j}=\{(t, z):(n-1) / n \leqslant t$ $\leqslant 1 ;(j-1) / n \leqslant z<j / n\}$. Let $\mu^{*}\left(Z_{i j}\right)=\omega_{i j}$. According to the definition $\sum_{j=1}^{n} \omega_{i j}=1 / n$. Let us subdivide interval $(i-1) / n \leqslant t \leqslant i / n$ into intervals

$$
\begin{gathered}
(i-1) / n \leqslant t \leqslant(i-1) / n+\omega_{i 1} \\
(i-1) / n+\omega_{i 1} \leqslant t \leqslant(i-1) / n+\omega_{i 1}+\omega_{i 2} ; \\
\ldots \\
\frac{i-1}{n}+\sum_{j=1}^{n-1} \omega_{i j} \leqslant t \leqslant \frac{i}{n}
\end{gathered}
$$

Let the function $x_{n}(t)$ equal $(k-1) / n$ on the interval

$$
\frac{i-1}{n}+\sum_{j=1}^{k-1} \omega_{i j} \leqslant t \leqslant \frac{i-1}{n}+\sum_{j=1}^{k} \omega_{i j}
$$

Let us define $x_{n}(t)$ in the same way on all the intervals $[(i-1) / n \leqslant t \leqslant i / n]$ and by means of that determine it on the whole interval $[0,1]$. The sequence of functions $x_{n}(t)$ satisfies the required condition.

Let $\psi(t, x)$ be a Lebesgue measurable function $(0 \leqslant t \leqslant 1,0 \leqslant x \leqslant 1,0 \leqslant$ $\psi(t, x) \leqslant 1)$. To every $\psi(t, x)$ there corresponds a measure $\mu_{\psi}$ on the unit square $Z$ defined by

$$
\mu_{\psi}(A)=\int_{0}^{1} \int_{0}^{1} \chi_{A}(t, \psi(t, x)) d t d x,
$$

where $A$ is any Borel set and $\chi_{A}(t, z)$ is a characteristic function of the set $A$. It can be easily checked that (1.7) holds for $\mu_{\psi}$ for all $\left[\alpha_{1}, \alpha_{2}\right] \subset[0,1]$ and thus $\mu_{\psi} \in M^{*}$.

Let $\mu^{*}$ be any measure from $M^{*}$. Let us fix a number $z_{0}, 0 \leqslant z_{0} \leqslant 1$. On Borel sets $B$ of the interval $[0,1]$ we define the countably additive measure $\mu_{z_{0}}$ by 


$$
\mu_{z_{0}}(B)=\mu^{*}\left\{(t, z): t \in B, z \leqslant z_{0}\right\} .
$$

Since $\mu^{*}$ satisfies (1.7), $\mu_{z_{0}}$ is absolutely continuous with respect to Lebesgue measure, and according to the Radon-Nikodym theorem a density $P_{z_{0}}(t)$ exists for the measure $\mu_{z_{0}}$ such that

$$
\mu_{z_{0}}(B)=\int_{B} P_{z_{0}}(t) d t
$$

We can assume that the functions $P_{z}(t)$ are defined except on sets of measure 0 and that $P_{z_{1}}(t) \leqslant P_{z_{2}}(t)$ for any $z_{1} \leqslant z_{2}$ and for all $t$, where both $P_{z_{1}}(t)$ and $P_{z_{2}}(t)$ are defined. Obviously $P_{1}(t)=1$. Let us now define the function

$$
\widetilde{\psi}(t, x)=\inf \left[z: P_{z}(t)=x\right] .
$$

If $\widetilde{\psi}(t, x)$ is not defined in $(t, x)$ by (1.9), then we set $\widetilde{\psi}(t, x)=\inf _{x<x^{\prime}} \widetilde{\psi}\left(t, x^{\prime}\right)$, where in $\left(t, x^{\prime}\right)$ the function $\widetilde{\psi}$ is defined by (1.9).

The measure $\mu_{\tilde{\psi}}$ is defined by (1.8), since $\widetilde{\psi}(t, x)$ is defined on the unit square and $0 \leqslant \widetilde{\psi}(t, x) \leqslant 1$. According to the definition we have

$$
\mu_{\tilde{\psi}}\left[(t, z): t \leqslant t_{0}, z \leqslant z_{0}\right]=\int_{0}^{t_{0}} P_{z_{0}}(t) d t=\mu^{*}\left[(t, z): t \leqslant t_{0}, z \leqslant z_{0}\right] .
$$

Hence the measures $\mu_{\tilde{\psi}}$ and $\mu^{*}$ are equal.

Note that two measures $\mu_{\psi_{1}}$ and $\mu_{\psi_{2}}$ are equal if and only if the joint distributions of the pair of functions $\left(t, \psi_{1}(t, x)\right)$ and $\left(t, \psi_{2}(t, x)\right)$ are equal.

Denote by $\Psi$ the set of all Lebesgue measurable functions $\psi(t, x)$ defined on the unit square $(0 \leqslant t \leqslant 1,0 \leqslant x \leqslant 1)$ and such that $0 \leqslant \psi(t, x) \leqslant 1$. The function $\psi_{1}$ is said to be equivalent to $\psi_{2}$ if the joint distributions of the pairs $\left(t, \psi_{1}(t, x)\right)$ and $\left(t, \psi_{2}(t, x)\right)$ are equal. We say that $\psi_{k}$ converges to $\psi$ if $\mu_{\psi_{k}}$ converges weakly to $\mu_{\psi}$. That means that $\psi_{k}$ converges to $\psi$, if for any $n=$ $0,1,2, \ldots$ and $m=1,2, \ldots$ we have

$$
\lim _{k \rightarrow \infty} \int_{0}^{1} \int_{0}^{1} t^{n} \psi_{k}^{m}(t, x) d t d x=\int_{0}^{1} \int_{0}^{1} t^{n} \psi^{m}(t, x) d t d x .
$$

We proved that $\Psi$ is a compact set homeomorphic to $M^{*}$ and thus to $g X$.

Let us denote the image of $\psi$ by the homeomorphism $\Psi$ on $g X$ by $a_{\psi}$. Consider the functional (1.6) $F_{n, m}(x)$. It is easy to see that

$$
F_{n, m}\left(a_{\psi}\right)=F_{n, m}\left(\mu_{\psi}\right)=\int_{0}^{1} \int_{0}^{1} t^{n} \psi^{m}(t, x) d t d x .
$$

Hence for the functional of integral form (0.2) we have

$$
\begin{aligned}
F\left(a_{\psi}\right) & =F\left(\mu_{\psi}\right) \\
& =\int_{0}^{1} \cdots \int_{0}^{1} \phi\left(t_{1}, \ldots, t_{k} ; \psi\left(t_{1}, x_{1}\right), \ldots, \psi\left(t_{k}, x_{k}\right)\right) d t_{1} \cdots d t_{k} d x_{1} \cdots d x_{k} .
\end{aligned}
$$

Thus we have the following basic result. 
THEOREM 1.2. Every point in $g X$ can be written as $a_{\psi}, \psi \in \Psi$. For any $\psi \in \Psi$ there exists $a_{\psi} \in g X$. The points $a_{\psi_{1}}$ and $a_{\psi_{2}}$ coincide if and only if the joint distributions of the pairs $\left(t, \psi_{1}(t, x)\right)$ and $\left(t, \psi_{2}(t, x)\right)$ are equal. The functional of integral form

$$
F(x)=\int_{0}^{1} \cdots \int_{0}^{1} \phi\left(t_{1}, \ldots, t_{k} ; x\left(t_{1}\right), \ldots, x\left(t_{k}\right)\right) d t_{1} \cdots d t_{k}
$$

has in $a_{\psi}$ the value

$$
F\left(a_{\psi}\right)=\int_{0}^{1} \cdots \int_{0}^{1} \phi\left(t_{1}, \ldots, t_{k} ; \psi\left(t_{1}, x_{1}\right), \ldots, \psi\left(t_{k}, x_{k}\right)\right) d t_{1} \cdots d t_{k} d x_{1} \cdots d x_{k} .
$$

Thus we have compactified the space $X$ of functions of one variable by the space $\Psi$ of functions of two variables.

2. Theory of integration. The generalization of integration from the probabilistic point of view was developed by $\mathrm{N}$. Wiener. His integration theory of the functionals, defined on random processes, is the infinite-dimensional generalization of Lebesgue integration theory, i.e. it defines the integration with respect to a countably additive measure, defined on an infinite-dimensional space, i.e. on a space of functions. Another approach to integration in functional spaces was proposed by R. Gâteaux in 1913-1914 (see [1]) and P. Levy in 1918-1919 (both of them belonged to Hadamard's school) and is less known. The GâteauxLevy integration theory was described in [2]. The integral is defined for functionals on infinite-dimensional space. The basic idea is as follows: first the average value of the functional on the $n$-dimensional unit ball (sphere) is evaluated and then the integral is defined as the limit of this average value for $n \rightarrow \infty$.

Further we consider only the functionals continuous in metric (0.1) of the space $X$. Our definition of integral being analogous to that of Gâteaux-Levy, we call our integral the Gâteaux-Levy integral. $\left({ }^{3}\right)$

Definition 2.1. Gateaux-Levy integral. Consider the points $0,1 / n, 2 / n$, $\ldots,(n-1) / n, 1$ on the interval $[0,1]$, where $n$ is a positive integer and consider the set $K_{n}$ of the functions from the space $X$ constant on the intervals $[(i-1) / n, i / n] \quad(i=1, \ldots, n)$. Every function $x(t) \in K_{n}$ is determined by a sequence of $n$ numbers $\left(x_{1}, \ldots, x_{n}\right) ; x_{i}$ being the value of $x(t)$ on $[(i-1) / n, i / n]$. The set $K_{n}$ can be considered as the $n$-dimensional unit cube, each point of it being a function. Let $F(x)$ be a continuous functional on $X$. This functional, considered on $K_{n}$, is a continuous function of $n$ variables $F^{(n)}\left(x_{1}, \ldots, x_{n}\right)$ defined on the unit cube. Let

$$
T_{n} F(x)=\int_{0}^{1} \cdots \int_{0}^{1} F^{(n)}\left(x_{1}, \ldots, x_{n}\right) d x_{1} \cdots d x_{n} .
$$

$\left({ }^{3}\right)$ The definition of Gâteaux-Levy integral in exactly the same form as ours was studied by different mathematicians. 
If there exists a finite $\lim _{n \rightarrow \infty} T_{n} F(x)=T F(x)$, then the functional $F(x)$ is said to be integrable, and the number $T F(x)$ is called its integral.

The Gâteaux-Levy integral has the following properties:

1. If the functionals $F_{1}(x)$ and $F_{2}(x)$ are integrable, then for any real numbers $d_{1}$ and $d_{2}$ the functional $d_{1} \cdot F_{1}(x)+d_{2} \cdot F_{2}(x)$ is also integrable, its integral being equal to $d_{1} \cdot T F_{1}(x)+d_{2} \cdot T F_{2}(x)$.

2. If the functional $F(x)$ is integrable and, for all functions $x \in X, F(x)$ $\geqslant 0$, then $T F(x) \geqslant 0$.

3. Let $F(x)$ be a functional and let there exist two integrable functionals $F_{1}^{(\epsilon)}(x)$ and $F_{2}^{(\epsilon)}(x)$ for any $\epsilon>0$ such that $F_{1}^{(\epsilon)}(x) \leqslant F(x) \leqslant F_{2}^{(\epsilon)}(x)$ and $T\left[F_{2}^{(\epsilon)}(x)-F_{1}^{(\epsilon)}(x)\right]<\epsilon$. Then the functional $F(x)$ is integrable.

4. Gâteaux formula. $\left({ }^{4}\right)$ The functional of integral form

$$
F(x)=\int_{0}^{1} \cdots \int_{0}^{1} \phi\left(t_{1}, \ldots, t_{k} ; x\left(t_{1}\right), \ldots, x\left(t_{k}\right)\right) d t_{1} \cdots d t_{k}
$$

is integrable, its integral being equal to

$$
T F=\int_{0}^{1} \cdots \int_{0}^{1} \phi\left(t_{1}, \ldots, t_{k} ; x_{1}, \ldots, x_{k}\right) d t_{1} \cdots d t_{k} d x_{1} \cdots d x_{k}
$$

Let $\psi(t, x) \in \Psi$, i.e. $\psi(t, x)$ be a Lebesgue measurable function on the unit square $(0 \leqslant t \leqslant 1,0 \leqslant x \leqslant 1)$ such that $0 \leqslant \psi(t, x) \leqslant 1$.

DEFINITION 2.2. Concept of the $\psi$-integral. Let $\psi_{i}^{(n)}(x)$ be a measurable function on $[0,1]$ such that its distribution function with respect to the Lebesgue measure on the interval is identical to the distribution function with respect to the Lebesgue measure on the unit square of the function $\psi(t \cdot n-i+1, x)$ $((i-1) / n \leqslant t \leqslant i / n, 0 \leqslant x \leqslant 1)$. Let $F(x)$ be a continuous functional on the space $X$. Let

$$
T_{\psi}^{(n)} F(x)=\int_{0}^{1} \cdots \int_{0}^{1} F^{(n)}\left(\psi_{1}^{(n)}\left(x_{1}\right), \ldots, \psi_{n}^{(n)}\left(x_{n}\right)\right) d x_{1} \cdots d x_{n},
$$

where $F^{(n)}\left(x_{1}, \ldots, x_{n}\right)$ is as in Definition 2.1. If there exists a finite $\lim _{n \rightarrow \infty} T_{\psi}^{(n)} F(x)=T_{\psi} F(x)$, then the functional is said to be $\psi$-integrable and the number $T_{\psi} F(x)$ is called its $\psi$-integral.

NoTE. The choice of functions $\psi_{1}^{(n)}, \ldots, \psi_{n}^{(n)}$ is obviously not unique, but this does not affect the definition of $T_{\psi}^{(n)} F(x)$.

Properties of the $\psi$-integral.

$1^{\prime}$. If the functionals $F_{1}(x)$ and $F_{2}(x)$ are $\psi$-integrable, then for any real numbers $d_{1}$ and $d_{2}$ the functional $d_{1} \cdot F_{1}(x)+d_{2} \cdot F_{2}(x)$ is $\psi$-integrable, its

$\left({ }^{4}\right)$ Formula (2.2) was obtained by Gâteaux (see [2]) in a slightly different form, since both the functional and the integral were considered not on the space $X$, but on the ball (sphere) in the infinite-dimensional space. A Gateaux formula in exactly the same form as ours was studied by different mathematicians. 
$\psi$-integral being equal to $d_{1} \cdot T_{\psi} F_{1}(x)+d_{2} \cdot T_{\psi} F_{2}(x)$.

$2^{\prime}$. If the functional $F(x)$ is $\psi$-integrable and for all $x \in X, F(x) \geqslant 0$, then $T_{\psi} F(x) \geqslant 0$.

$3^{\prime}$. Let $F(x)$ be a functional and let there exist two $\psi$-integrable functionals $F_{1}^{(\epsilon)}(x)$ and $F_{2}^{(\epsilon)}(x)$ for any $\epsilon>0$ such that

$$
F_{1}^{(\epsilon)}(x) \leqslant F(x) \leqslant F_{2}^{(\epsilon)}(x) \text { and } T_{\psi}\left[F_{2}^{(\epsilon)}(x)-F_{1}^{(\epsilon)}(x)\right]<\epsilon .
$$

Then the functional $F(x)$ is $\psi$-integrable.

$4^{\prime}$. The functional $F(x)$ of integral form (2.1) is $\psi$-integrable and

(2.3) $T_{\psi} F=\int_{0}^{1} \cdots \int_{0}^{1} \phi\left(t_{1}, \ldots, t_{k} ; \psi\left(t_{1}, x_{1}\right), \ldots, \psi\left(t_{k}, x_{k}\right)\right) d t_{1} \cdots d t_{k} d x_{1} \cdots d x_{k}$.

Evidently the notion of the $\psi$-integral is the generalization of the notion of the Gâteaux-Levy integral. For $\psi(t, x)=x$ the $\psi$-integral is the Gâteaux-Levy integral. Properties 1', 2', $3^{\prime}$ are obvious; property $4^{\prime}$ can be checked directly. If the joint distributions of the pairs of functions $t, \psi_{1}(t, x)$ and $t, \psi_{2}(t, x)$ are equal, then a $\psi_{1}$-integrable functional $F(x)$ is also $\psi_{2}$-integrable and $T_{\psi_{1}} F(x)=T_{\psi_{2}} F(x)$.

Let us remember that the equalities (1.1)-(1.4) and Theorem 1.1 imply the fact that the algebra of functionals of integral form have uniform closure $G(X)$ and that $G(X)$ determines a metric compactification $g X$ of $X$. By virtue of Theorem 1.2 and properties of the $\psi$-integral, for any $F(x) \in G(X)$ and for any $\psi \in \Psi$ we have $T_{\psi} F(x)=F\left(a_{\psi}\right)$. For the same reason any linear multiplicative functional continuous on $G(X)$ with its value on the functional 1 being equal to 1 is a $\psi$-integral.

Note that we can obtain the principal properties of the $\psi$-integral from $1^{\prime}$, $2^{\prime}, 3^{\prime}, 4^{\prime}$. For example, multiplicativity holds for the functionals of integral form and thus for all functionals of $G(X)$. In fact, the equalities (1.1), (1.2), (1.4) and property $4^{\prime}$ imply

$$
\begin{aligned}
& T_{\psi}\left[F_{1}(x) \cdot F_{2}(x)\right] \\
& =\int_{0}^{1} \cdots \int_{0}^{1} \phi_{1}\left(t_{1}, \ldots, t_{k_{1}} ; \psi\left(t_{1}, x_{1}\right), \ldots, \psi\left(t_{k_{1}}, x_{k_{1}}\right)\right) \\
& \quad \cdot \phi_{2}\left(t_{k_{1}+1}, \ldots, t_{k_{1}+k_{2}} ; \psi\left(t_{k_{1}+1}, x_{k_{1}+1}\right), \ldots, \psi\left(t_{k_{1}+k_{2}}, x_{k_{1}+k_{2}}\right)\right) \\
& \quad d t_{1} \cdots d t_{k_{1}+k_{2}} d x_{1} \cdots d x_{k_{1}+k_{2}} \\
& =\int_{0}^{1} \cdots \int_{0}^{1} \phi_{1}\left(t_{1}, \ldots, t_{k_{1}} ; \psi\left(t_{1}, x_{1}\right), \ldots, \psi\left(t_{k_{1}}, x_{k_{1}}\right)\right) d t_{1} \cdots d t_{k_{1}} d x_{1} \cdots d x_{k_{1}} \\
& \quad \times \int_{0}^{1} \cdots \int_{0}^{1} \phi_{2}\left(t_{1}, \ldots, t_{k_{2}} ; \psi\left(t_{1}, x_{1}\right), \ldots, \psi\left(t_{k_{2}}, x_{k_{2}}\right)\right) d t_{1} \cdots d t_{k_{2}} d x_{1} \cdots d x_{k_{2}} \\
& =T_{\psi} F_{1}(x) \cdot T_{\psi} F_{2}(x) .
\end{aligned}
$$


Consider the Gâteaux-Levy integral. Since the Gateaux-Levy integral is the $\psi$ integral for $\psi(t, x)=x$, the Gâteaux-Levy integral of a functional $F(x) \in G(X)$ is $T F(x)=F\left(a_{x}\right)$. From the first section of the present paper it follows that $a_{\psi}$, and in particular $a_{x}$, belong to $g X \backslash X$ if $\psi \in \Psi \backslash X$. Thus the Gâteaux-Levy integral on $G(X)$ generates a measure on $g X$ concentrated at the point $a_{x} \in g X \backslash X$, and hence the Gâteaux-Levy integral does not generate a countable additive measure on $X .\left({ }^{5}\right)$

Let $U\left(a_{x}\right)$ be an open neighbourhood of $a_{x}$ in $g X$ and $U^{\prime}=X \cap U$. According to Definition 2.1 of the Gâteaux-Levy integral we have $\lim _{n \rightarrow \infty} \mu_{n}\left(U^{\prime} \cap K_{n}\right)=1$, where $K_{n}$ is the set from Definition 2.1 which can be identified with the $n$-dimensional unit cube, and $\mu_{n}$ is the Lebesgue measure on this cube. Now we describe in our own terms the following phenomenon, first investigated by Borel (see [3]) and then by Gâteaux, Levy (see [2]) and other mathematicians: for any functional $F(x)$ of integral form (2.1) and any $\epsilon>0$ there exists an open set $V^{\prime} \subset X$ such that

$$
\lim _{n \rightarrow \infty} \mu_{n}\left(K_{n} \cap V^{\prime}\right)=1
$$

and for any point $x^{\prime} \in V^{\prime}$ we have $\left|F\left(x^{\prime}\right)-T F(x)\right|<\epsilon$. The proof is very simple. Consider $F(x)$ on $g X$. The open set $V \subset g X$ consists of those points $x \in g X$ for which $\left|F\left(a_{x}\right)-F(x)\right|<\epsilon$. The set $V^{\prime}=V \cap X$ satisfies all the conditions because $F\left(a_{x}\right)=T F(x)$.

Let us define the set $L(X)$ to be the set of all bounded continuous functionals $F(x)$ such that for any $\epsilon>0$ there exist $G_{1}(x), G_{2}(x) \subset G(X)$ such that $G_{1}(x) \leqslant F(x) \leqslant G_{2}(x)$ and $T\left[G_{2}(x)-G_{1}(x)\right]<\epsilon$. According to property 3 of the Gâteaux-Levy integral the functional $F(x)$ is integrable.

Let $\beta X$ be the Čch compactification of $X$. It is a maximal compactification of $X$ and every bounded continuous functional on $X$ can be continuously extended on $X$. Consider the natural mapping of $\beta X$ on $g X$. We denote the preimage of the point $a_{x}$ by $A_{x}$. If $F(x) \in L(X)$, then $F(x)$ is constant on $A_{x}$, its value on $A_{x}$ being the value of $T F(x)$. On the other hand any functional $F(x)$ constant and equal to $d$ on $A_{x}$ belongs to $L(X)$. In fact, for $\epsilon>0$ let $U^{\prime}$ be the open set of all $x^{\prime} \in \beta X$ such that $\left|F\left(x^{\prime}\right)-d\right|<\epsilon$. Let $\phi^{\prime}=\beta X-U^{\prime}$ and let $\phi$ be the image of $\phi^{\prime}$ in the natural mapping of $\beta X$ on $g X$. If we set $U=g X \backslash \phi$, then $a_{x} \in U$. There are functionals $G_{1}(x), G_{2}(x) \subset G(X)$ such that

if $x \in \phi$ and

$$
G_{1}\left(a_{x}\right)=d-\epsilon, \quad G_{1}(x)=\min \left[d-\epsilon, \min _{x^{\prime} \in \beta X} F\left(x^{\prime}\right)\right]=F_{*},
$$

${ }^{5}$ ) The fact that the Gâteaux-Levy integral does not generate a countably additive measure also follows from the ideas described in [2]. 
$F_{*} \leqslant G_{1}(x) \leqslant d-\epsilon ; \quad G_{2}\left(a_{x}\right)=d+\epsilon, \quad G_{2}(x)=\max \left[d+\epsilon, \max _{x^{\prime} \in \beta X} F\left(x^{\prime}\right)\right]=F^{*}$,

if $x \in \phi$ and

$$
F^{*} \geqslant G_{2}(x) \geqslant d+\epsilon .
$$

Obviously $G_{1}(x) \leqslant F(x) \leqslant G_{2}(x)$ and $T\left[G_{2}(x)-G_{1}(x)\right]=2 \epsilon$. By definition $F(x) \in L(X)$.

Let $B$ be a closed set in $\beta X$ with the property that every bounded continuous functional constant on $B$ is integrable, its Gâteaux-Levy integral being equal to its value on $B$. For example, a closed set $B \supset A_{x}$ is such a set. It can be easily proved that for any set $U \supset B, U$ open in $\beta X$, we have

$$
\lim _{n \rightarrow \infty} \mu_{n}\left(K_{n} \cap U\right)=1
$$

On the other hand, if for a closed set $B \subset \beta X$ we have $\lim _{n \rightarrow \infty} \mu_{n}\left(K_{n} \cap U\right)=1$ for every open set $U \supset B$, then any functional constant on $B$ is integrable, its Gâteaux-Levy integral being equal to its value on $B$. If each of a finite number of closed sets $B_{1}, \ldots, B_{n}$ has the same property as the set $B$, then the set $\bigcap_{i=1}^{n} B_{i} \neq \varnothing$ and it has the same property. Thus if $\left\{B_{\alpha}\right\}$ is the family of all closed sets, having the property of the set $B$, then the set $\bigcap_{\alpha} B_{\alpha}=B_{x} \neq \varnothing$ and the set $B_{x}$ has the property of the set $B$. It is clear that integrability or nonintegrability of a functional depends only on its behavior in a neighborhood of the set $B_{x}$.

Definition 2.3. A bounded continuous functional constant on $B_{x}$ is said to be a functional constant.

The set of all functional constants is denoted by $L_{x}(X)$. This definition is reasonable, since for any bounded continuous and integrable functional $F(x)$ and for any functional $L(x) \in L_{x}(X)$ the functional $F(x) \cdot L(x)$ is integrable and $T[F(x) \cdot L(x)]=T F(x) \cdot T L(x)$. A bounded continuous functional $L(x)$ belongs to $L_{x}(X)$ if and only if $[T L(x)]^{2}=T L^{2}(x)$, assuming that both $T L(x)$ and $T L^{2}(x)$ exist.

The set $A_{x}$ is a $G_{\delta}$ set, i.e. there exists in $\beta X$ a system of open sets $U_{1} \supset$ $U_{2} \supset \cdots U_{n} \supset \cdots$ such that $\bigcap_{n=1}^{\infty} U_{n}=A_{x}$. This follows from the fact that $A_{x}$ is a pre-image of a point in the natural mapping of $\beta X$ on the compact metric space $g X$.

THEOREM 2.1. The set $B_{x}$ is not a $G_{\delta}$ set.

Proof. In Definition 2.1 we considered the sets $K_{n}$ of functions constant on the intervals $[0,1 / n],[1 / n, 2 / n], \ldots,[(n-1) / n, 1]$. Each function in $K_{n}$ is determined by $n$ numbers $x_{1}, x_{2}, \ldots, x_{n}$, the values of this function on the 
respective intervals. Every function in $K_{n}$ is a point of the $n$-dimensional unit cube and vice versa. Consider the set in the $n$-dimensional unit cube $(n \geqslant 2)$

$$
X_{n}^{\prime}=\left\{\left(x_{1}, \ldots, x_{n}\right):\left|x_{1}-x_{2}\right|+\left|x_{3}-x_{4}\right|+\cdots+\left|x_{n-2}-x_{n-1}\right| \geqslant 1 / 2(n-1) \cdot p\right\},
$$

if $n$ is an odd number and

$$
X_{n}^{\prime}=\left\{\left(x_{1}, \ldots, x_{n}\right):\left|x_{1}-x_{2}\right|+\left|x_{3}-x_{4}\right|+\cdots+\left|x_{n-1}-x_{n}\right| \geqslant 1 / 2 n \cdot p\right\},
$$

if $n$ is even; $p$ is fixed and $0<p<1 / 3$. The set $X_{1}^{\prime}=K_{1}$. According to the law of large numbers $\lim _{n \rightarrow \infty} \mu_{n}\left(X_{n}^{\prime}\right)=1$, where $\mu_{n}$ is the Lebesgue measure of the $n$-dimensional unit cube, since $\int_{0}^{1} \int_{0}^{1}\left|x_{i}-x_{i+1}\right| d x_{i} d x_{i+1}=1 / 3$. The sets $X_{1}^{\prime}, \ldots, X_{n}^{\prime}, \ldots$ are closed in $X$ and also closed in $X$ are the sets $\bigcup_{i=1}^{\infty} X_{n_{i}}^{\prime}$, where $n_{1}, \ldots, n_{i}, \ldots$ is any sequence of positive integers. There exist closed sets $X_{n} \subset X_{n}^{\prime}$ such that $\lim _{n \rightarrow \infty} \mu_{n}\left(X_{n}\right)=1$ and $X_{i} \neq X_{j}$ for $i \neq j$. Suppose that there exists in $\beta X$ a sequence of open sets

$$
U_{1} \supset U_{2} \supset \cdots U_{m} \supset \cdots \text { and } \bigcap_{m=1}^{\infty} U_{m}=B_{x} \text {. }
$$

Let us take a decreasing sequence of numbers $\epsilon_{1}>\epsilon_{2}>\cdots>\epsilon_{m}>\cdots$ such that $\epsilon_{m} \rightarrow 0$. For $\epsilon_{1}$ there exists a positive integer $n_{1}$ such that for all $n \geqslant n_{1}$ we have $\mu_{n}\left(X_{n} \cap U_{1}\right) \geqslant 1-\epsilon_{1}$. For $\epsilon_{2}$ there exists a positive integer $n_{2}>n_{1}$ such that for all $n \geqslant n_{2}$ we have $\mu_{n}\left(X_{n} \cap U_{2}\right) \geqslant 1-\epsilon_{2}$. Similarly there exist numbers $n_{3}<n_{4}<\cdots<n_{m}<\cdots$. Consider the sets

$$
\begin{aligned}
& U^{\left(n_{1}\right)}=X_{n_{1}} \cap U_{1} ; U^{\left(n_{1}+1\right)}=X_{n_{1}+1} \cap U_{1} ; \ldots ; U^{\left(n_{2}-1\right)}=X_{n_{2}-1} \cap U_{1} ; \\
& U^{\left(n_{2}\right)}=X_{n_{2}} \cap U_{2} ; U^{\left(n_{2}+1\right)}=X_{n_{2}+1} \cap U_{2} ; \ldots ; U^{\left(n_{3}-1\right)}=X_{n_{3}-1} \cap U_{2} ; \\
& \ldots . \\
& U^{\left(n_{m}\right)}=X_{n_{m}} \cap U_{m} ; U^{\left(n_{m}+1\right)}=X_{n_{m}+1} \cap U_{m} ; \ldots ; U^{\left(n_{m+1}-1\right)}=X_{n_{m+1}-1} \cap U_{m} .
\end{aligned}
$$

Set $U^{(1)}=X_{1}, U^{(2)}=X_{2}, \ldots, U^{\left(n_{1}-1\right)}=X_{n_{1}-1}$. If $\left\{x_{n}\right\}$ is a sequence of functions such that for any $n, x_{n} \in U^{(n)}$, then the closure of the set $\left\{x_{n}\right\}$ in $\beta X$ is a closed set $\widetilde{X}_{0}$ and $\tilde{X}_{0} \backslash\left\{x_{n}\right\} \subset B_{x}$. Every function $x_{n} \in X_{n}$ and so $\rho_{n}=$ $\rho\left(x_{n}, \cup_{i \neq n} X_{i}\right)>0$. Let $V_{n}$ be the $\rho_{n}^{\prime}$-neighborhood of the function $x_{n}$. For numbers $\rho_{n}^{\prime}$ the following conditions hold:

(a) $0<\rho_{n}^{\prime}<\rho_{n} / 4$,

(b) $\lim _{n \rightarrow \infty} \mu_{n}\left(V_{n} \cap K_{n}\right)=0$.

There exists a continuous functional $\widetilde{L}_{0}(x)$ for which

(1) $0 \leqslant \widetilde{L}_{0}(x) \leqslant 1$,

(2) $\widetilde{L}_{0}\left(x_{n}\right)=0$ for every $n$,

(3) $\widetilde{L}_{0}(x)=1$, if $x \in X \backslash \bigcup_{n=1}^{\infty} V_{n}$. 
The functionals $\tilde{L}_{0}(x)$ and $\tilde{L}_{0}^{2}(x)$ are integrable and $T \widetilde{L}_{0}(x)=T \widetilde{L}_{0}^{2}(x)=1$. So for $x_{0} \in B_{x}, \widetilde{L}_{0}\left(x_{0}\right)=1$. And also for every point $x_{0} \in \widetilde{X}_{0} \backslash\left\{x_{n}\right\} \subset B_{x}, \widetilde{L}_{0}\left(x_{0}\right)$ $=0$. The contradiction received proves the theorem. Q.E.D.

It is obvious that $B_{x} \subset A_{x}$. Theorem 2.1 implies that $B_{x} \neq A_{x}$. Denote the pre-image of the point $a_{\psi}$ under natural mapping of $\beta X$ onto $g X$ by $A_{\psi}$. The Banach algebra $L_{\psi}(X)$ of $\psi$-functional constants is the set of all bounded continuous functionals $F(x)$ such that $\left[T_{\psi} F(x)\right]^{2}=T_{\psi} F^{2}(x)$, assuming that both $T_{\psi} F(x)$ and $T_{\psi} F^{2}(x)$ exist. If $F(x) \in L_{\psi}(X)$ and $\hat{F}(x)$ is a bounded continuous $\psi$-integrable functional, then $T_{\psi}[F(x) \cdot \hat{F}(x)]=T_{\psi} F(x) \cdot T_{\psi} \hat{F}(x)$. There exists a closed set $B_{\psi} \subset A_{\psi}$ such that $F(x) \in L_{\psi}(X)$ if and only if $F(x)$ is constant on $B_{\psi}$. Existence or nonexistence of the $\psi$-integral depends only on the behaviour of the functional in a neighbourhood of the set $B_{\psi}$. The set $A_{\psi}$ is a $G_{\delta}$ set. If $\psi \in \Psi \backslash X$ then $B_{\psi}$ is not a $G_{\delta}$ set and thus $B_{\psi} \neq A_{\psi}$. If $\psi(t, x)=x_{0}(t) \in X$, then $T_{\psi} F(x)=F\left(x_{0}(t)\right)$ and $A_{\psi}=B_{\psi}=x_{0}(t)$. By virtue of Theorem 1.2, $\beta X$ is the union of sets $A_{\psi}$.

EXAMPLE 2.1. The functional $Q(x)=\int_{0}^{1} x(t) x(t+\tau) d t$, where $\tau$ is a fixed number, $0<\tau<1$. The sum $t+\tau$ is considered modulo 1. For $\psi \in \Psi$ one evaluates immediately

$$
\begin{aligned}
T_{\psi} Q(x) & =\int_{0}^{1} \int_{0}^{1} \int_{0}^{1} \psi(t, x) \psi(t+\tau, y) d t d x d y, \\
Q^{2}(x) & =\int_{0}^{1} \int_{0}^{1} x\left(t_{1}\right) x\left(t_{2}\right) x\left(t_{1}+\tau\right) x\left(t_{2}+\tau\right) d t_{1} d t_{2} .
\end{aligned}
$$

Also directly we get

$$
\begin{aligned}
T_{\psi} Q^{2}(x) & =\int_{0}^{1} \int_{0}^{1} \int_{0}^{1} \int_{0}^{1} \int_{0}^{1} \int_{0}^{1} \psi\left(t_{1}, x_{1}\right) \psi\left(t_{2}, x_{2}\right) \psi\left(t_{1}+\tau, y_{1}\right) \psi\left(t_{2}+\tau, y_{2}\right) d t_{1} d t_{2} d x_{1} d x_{2} d y_{1} d y_{2} \\
& =\left[\int_{0}^{1} \int_{0}^{1} \int_{0}^{1} \psi(t, x) \psi(t+\tau, y) d t d x d y\right]^{2}=\left[T_{\psi} Q(x)\right]^{2} .
\end{aligned}
$$

Thus for any $\psi \in \Psi$ the functional $Q(x) \in L_{\psi}(X)$, i.e. it is constant on $B_{\psi}$. Let a sequence of functions $x_{k}(t)$ converge in $g X$ to a point $a_{\psi}$. That means that for any $n=0,1,2, \ldots$ and $m=1,2, \ldots$ we have

$$
\lim _{k \rightarrow \infty} \int_{0}^{1} t^{n} x_{k}^{m}(t) d t=\int_{0}^{1} \int_{0}^{1} t^{n} \psi^{m}(t, x) d t d x .
$$

For any $\psi \in \Psi \backslash X$ one can easily get a sequence $x_{k}(t)$ converging in $g X$ to $a_{\psi}$ such that the limit $\lim _{k \rightarrow \infty} Q\left(x_{k}(t)\right)$ does not exist. That means that, in spite of the fact that $Q(x)$ is constant on $B_{\psi}$ for any $\psi \in \Psi$, the functional $Q(x)$ is not constant on $A_{\psi}$ for any $\psi \in \Psi \backslash X$.

The investigation of $\psi$-integral by means of compactifications gives some nontrivial properties of the functionals of the space $X$. Let $F(x)$ be a bounded continuous functional and let 


$$
F^{(n)}\left(\psi_{1}^{(n)}\left(x_{1}\right), \ldots, \psi_{n}^{(n)}\left(x_{n}\right)\right)
$$

be the functions introduced in Definition 2.2. Let

$$
\begin{aligned}
& \varlimsup_{n \rightarrow \infty} \int_{0}^{1} \cdots \int_{0}^{1} F^{(n)}\left(\psi_{1}^{(n)}\left(x_{1}\right), \ldots, \psi_{n}^{(n)}\left(x_{n}\right)\right) d x_{1} \cdots d x_{n}=\bar{T}_{\psi} F(x), \\
& \varliminf_{n \rightarrow \infty} \int_{0}^{1} \cdots \int_{0}^{1} F^{(n)}\left(\psi_{1}^{(n)}\left(x_{1}\right), \ldots, \psi_{n}^{(n)}\left(x_{n}\right)\right) d x_{1} \cdots d x_{n}=\underline{T}_{\psi} F(x) .
\end{aligned}
$$

If $\bar{T}_{\psi} F(x)=\underline{T}_{\psi} F(x)$, then by virtue of Definition 2.2, $F(x)$ is $\psi$-integrable.

THEOREM 2.2. If $F(x)$ is a bounded continuous functional $\psi \in \Psi$, and $d$ is a fixed number such that $\bar{T}_{\psi} F(x) \geqslant d \geqslant I_{\psi} F(x)$, then there exist:

(1) A sequence of functions $\left\{\psi_{k}\right\} \subset \Psi$ converging to $\psi$, i.e. for any $n=$ $0,1,2, \ldots$ and $m=1,2, \ldots$ we have

$$
\lim _{k \rightarrow \infty} \int_{0}^{1} \int_{0}^{1} t^{n} \psi_{k}^{m}(t, x) d t d x=\int_{0}^{1} \int_{0}^{1} t^{n} \psi^{m}(t, x) d t d x .
$$

(2) Two sequences of functionals of integral form $G_{11}, G_{21}, \ldots, G_{k 1}, \ldots$, $G_{12}, G_{22}, \ldots, G_{k 2}, \ldots$ such that for all $k, G_{k 1}(x) \leqslant F(x) \leqslant G_{k 2}(x)$ and

$$
\lim _{k \rightarrow \infty} T_{\psi_{k}} G_{k 1}(x)=\lim _{k \rightarrow \infty} T_{\psi_{k}} G_{k 2}(x)=d .
$$

One can arrange that $\left\{\psi_{k}\right\} \subset \Psi \backslash X$.

Proof. (1) The set $A_{\psi}$ is connected. Consider the system of functions defined on $[0,1]$ :

$$
\begin{aligned}
& \varphi_{11} \\
& \varphi_{12} ; \varphi_{22} \\
& \varphi_{1,2^{n}}^{\bullet} ; \varphi_{2,2^{n}} ; \ldots ; \varphi_{2^{n}, 2^{n}}
\end{aligned}
$$

where $\varphi_{k, 2^{n}}$ is equal to 1 on the interval $\left[(k-1) / 2^{n}, k / 2^{n}\right]$ and vanishes outside this interval. Obviously, the functionals of the form

$$
\widetilde{F}(x)=\int_{0}^{1} \varphi_{k, 2^{n}}(t) x^{m}(t) d t,
$$

where $m$ is any positive integer, generate an algebra, its uniform closure being $G(X)$. That means that for any point $a_{\psi} \in g X$ and for any neighbourhood $U\left(a_{\psi}\right)$ in $g X$, there exists a finite set of functionals of the form $(2.4), \widetilde{F}_{1}(x), \ldots, \widetilde{F}_{q}(x)$, and a finite set of positive numbers $\epsilon_{1}, \ldots, \epsilon_{q}$ such that the set

$$
V=\left\{x:\left|\widetilde{F}_{1}(x)-\widetilde{F}_{1}\left(a_{\psi}\right)\right|<\epsilon_{1}, \ldots,\left|\widetilde{F}_{q}(x)-\widetilde{F}_{q}\left(a_{\psi}\right)\right|<\epsilon_{q}\right\}
$$

is open in $g X$, contains the point $a_{\psi}$ and $V \subset U\left(a_{\psi}\right)$. Suppose that the set $A_{\psi}$ is not connected. Then there exist in $\beta X$ two open sets $U_{1}$ and $U_{2}, U_{1} \cup U_{2} \supset A_{\psi}$, 
$\bar{U}_{1} \cap \bar{U}_{2}=\varnothing$, where $\bar{U}_{1}, \bar{U}_{2}$ are closures of $U_{1}$ and $U_{2}$ in $\beta X$. Let $U_{1} \cap X=$ $V_{1}$ and $U_{2} \cap X=V_{2}$. Since $A_{\psi}$ is the pre-image of the point $a_{\psi}$ under the natural mapping of $\beta X$ onto $g X$, then there exists in $g X$ a neighborhood $U\left(a_{\psi}\right)$ of $a_{\psi}$ such that $U\left(a_{\psi}\right) \cap X \subset V_{1} \cup V_{2}$. According to previous arguments there exists in $X$ an open set

$$
\begin{array}{r}
V=\left\{x(t):\left|\int_{0}^{1} \varphi_{k_{1}, 2} n_{1}(t) x^{m_{1}}(t) d t-\int_{0}^{1} \int_{0}^{1} \varphi_{k_{1}, 2^{n_{1}}}(t) \psi^{m_{1}}(t, x) d t d x\right|<\epsilon_{1}, \ldots,\right. \\
\left.\left|\int_{0}^{1} \varphi_{k_{q}, 2^{n_{q}}}(t) x^{m_{q}}(t) d t-\int_{0}^{1} \int_{0}^{1} \varphi_{k_{q}, 2^{n_{q}}}(t) \psi^{m_{q}}(t, x) d t d x\right|<\epsilon_{q}\right\}
\end{array}
$$

such that both $V_{1}$ and $V_{2}$ contain points of $V$ and $V \subset V_{1} \cup V_{2}$. Hence the set $V$ is disconnected. Note that the indices $n_{1}, \ldots, n_{q}$ of the functions $\varphi_{k_{1}, 2^{n 1}} ; \ldots ; \varphi_{k_{q}, 2^{n}}$ in the definition of the set $V$ are, generally speaking, distinct. One can easily make them equal. For any two functions $x_{1}(t), x_{2}(t) \subset V$ there exists a continuous mapping $\pi$ of the interval $[0,1]$ into $V$ such that $\pi(0)$ $=x_{1}(t), \pi(1)=x_{2}(t)$. This contradiction proves that the set $A_{\psi}$ is connected.

(2) Set $\bar{M}=\max _{x \in A_{\psi}} F(x), \underline{M}=\min _{x \in A} F(x)$. It can be easily proved that $\bar{M} \geqslant \bar{T}_{\psi} F(x) \geqslant I_{\psi} F(x) \geqslant \underline{M}$. The set $A_{\psi}$ being connected, there exists a point $x_{0} \in A_{\psi}$ such that $F\left(x_{0}\right)=d$. Consider the sets $U_{1}, U_{2}, \ldots, U_{k}, \ldots$ in $X$, where

$$
U_{k}=\{x(t):|F(x(t))-d|<1 / k\} .
$$

Let $V_{1} \supset V_{2} \supset \cdots \supset V_{k} \supset \cdots$ be a sequence of open sets in $g X$ such that $\bigcap_{k=1}^{\infty} V_{k}=a_{\psi}$. Consider the open sets in $X$ :

$$
W_{1}=U_{1} \cap V_{1}, \ldots, W_{k}=U_{k} \cap V_{k}, \ldots
$$

These sets are nonempty by construction.

Let $W_{k}^{\prime}$ be open sets in $g X$ such that $W_{k}^{\prime} \cap X=W_{k}$ and $W_{k}^{\prime} \subset V_{k}$. Let us select a point $a_{\psi_{k}}$ in each of them. Obviously we can select them so that $a_{\psi_{k}} \in g X \backslash X$. The sequence of functions $\psi_{k}$ converges to $\psi$. Set

$$
\max _{x \in A_{\psi_{k}}} F(x)+\frac{1}{2^{k}}=M^{(k)}, \quad \min _{x \in A_{\psi_{k}}} F(x)-\frac{1}{2^{k}}=M_{(k)} .
$$

We can construct two sequences of functionals in $G(X)$

$$
\begin{aligned}
& G_{11}^{\prime}, G_{21}^{\prime}, \ldots, G_{k 1}^{\prime}, \ldots, \\
& G_{12}^{\prime}, G_{22}^{\prime}, \ldots, G_{k 2}^{\prime}, \ldots,
\end{aligned}
$$

so that for all $k$ we have

$$
G_{k 1}^{\prime}(x) \leqslant F(x) \leqslant G_{k 2}^{\prime}(x), \quad T_{\psi_{k}} G_{k 1}^{\prime}=M_{(k)}, \quad T_{\psi_{k}} G_{k 2}^{\prime}=M^{(k)} .
$$


However $\lim _{k \rightarrow \infty} M_{(k)}=\lim _{k \rightarrow \infty} M^{(k)}=d$.

Note now that any functional in $G(x)$ can be uniformly approximated by the functionals of integral form, and thus sequences (2.5) and (2.6) can be replaced by similar sequences of functionals of integral form satisfying the assertion of the theorem. Q.E.D.

The fact that $A_{\psi}$ is a connected set is very interesting, since $B_{\psi}$ is disconnected for any $\psi \in \Psi \backslash X$.

3. Integrable and nonintegrable functionals. In the proof of Theorem 2.1 we introduced the sets $X_{n} \subset X$. The sets $\bigcup_{i=1}^{\infty} X_{2 i-1}$ and $\bigcup_{i=1}^{\infty} X_{2 i}$ are closed and $\left[\bigcup_{i=1}^{\infty} X_{2 i-1}\right] \cap\left[\bigcup_{i=1}^{\infty} X_{2 i}\right]=\varnothing$.

EXAMPLE 3.1. A bounded continuous functional $K(x)$ which is not GateauxLevy integrable. Let $K(x)$ be equal to 1 on $\bigcup_{i=1}^{\infty} X_{2 i-1}$ and vanish on $\bigcup_{i=1}^{\infty} X_{2 i}$. Obviously this functional is not integrable.

EXAMPLE 3.2. A bounded continuous Gateaux-Levy integrable functional $S(x)$ such that $S^{2}(x)$ is not Gâteaux-Levy integrable. As we know $X_{n}$ can be considered as a subset of the $n$-dimensional unit cube and $\lim _{n \rightarrow \infty} \mu_{n}\left(X_{n}\right)=1$, where $\mu_{n}$ is the Lebesgue measure on the $n$-dimensional unit cube. If $n$ is an odd number, we take a function $f\left(x_{1}, \ldots, x_{n}\right)=x_{1}$, where $\left(x_{1}, \ldots, x_{n}\right)$ are coordinates of the point in the $n$-dimensional unit cube. We denote this function on $X_{n} \subset X$ by $f_{n}$. If $n$ is an even number, we take the function $g\left(x_{1}, \ldots, x_{n}\right)=$ $3 x_{1}^{2} / 2$ on the $n$-dimensional unit cube. This function on $X_{n}$ we denote by $g_{n}$. Let us take a bounded continuous functional on $X$ equal to $f_{n}$ on $X_{n}$ for odd $n$ and $g_{n}$ on $X_{n}$ for even $n$. By virtue of Definition 2.1 of the Gâteaux-Levy integral this functional is integrable and $T S(x)=1 / 2$, whilst the functional $S^{2}(x)$ is not integrable.

In Definition 1.1 of the Gâteaux-Levy integral we considered the $n$-dimensional unit cube $K_{n}$ and the function $F^{(n)}\left(x_{1}, \ldots, x_{n}\right)$ induced by the functional $F(x)$ on $K_{n}$. Let $m$ functionals $F_{1}(x), \ldots, F_{m}(x)$ be given; to each of these functionals corresponds a function on $K_{n}$ :

$$
F_{1}^{(n)}\left(x_{1}, \ldots, x_{n}\right), \ldots, F_{m}^{(n)}\left(x_{1}, \ldots, x_{n}\right) .
$$

Denote by $f_{m}^{(n)}$ the joint distribution of functions (3.1) with respect to Lebesgue measure on the $n$-dimensional unit cube.

Let $A(X)$ be a Banach algebra of bounded continuous functionals. Each functional belonging to $A(X)$ is integrable if and only if there exists a family of functionals $\left\{F_{\alpha}\right\} \subset A(X)$ generating an algebra whose uniform closure is $A(X)$ and such that for any finite number of functionals $F_{1}, \ldots, F_{m} \subset\left\{F_{\alpha}\right\}$ their joint distributions $f_{m}^{(n)}$ converge weakly for $n \rightarrow \infty$.

Let $F_{\max }(X)$ be a maximal algebra of bounded continuous Gâteaux-Levy 
integrable functionals, i.e. an algebra such that there is no algebra $A(X) \supset$ $F_{\max }(X)\left(A(X) \backslash F_{\max }(X) \neq \varnothing\right)$ consisting only of bounded continuous GâteauxLevy integrable functionals. Evidently $F_{\max }(X) \supset L_{x}(X)$, the space of all functionals constants (Definition 2.3).

THEOREM 3.1. The intersection of all maximal algebras of bounded continuous Gâteaux-Levy integrable functionals is $L_{x}(X)$.

Proof. Denote the intersection of all $F_{\max }(X)$ by $L_{\max }(X)$. Let $F(x) \in$ $L_{\max }(X)$ and suppose that $F(x) \notin L_{x}(X)$. Denote by $f^{(n)}$ the distribution of the function $F^{(n)}\left(x_{1}, \ldots, x_{n}\right)$ induced by $F(x)$ on $K_{n}$. For $n \rightarrow \infty, f^{(n)}$ converges weakly to a distribution function $f^{(0)}$. There exists a Lebesgue measurable function $\varphi$ defined on $[0,1]$ such that its distribution function with respect to Lebesgue measure on $[0,1]$ is equal to $f^{(0)}$. Since $F(x) \notin L_{x}(X), \varphi$ is not constant on a set of measure 1 in the interval $[0,1]$. We can find continuous functions $F_{*}^{(n)}\left(x_{1}, \ldots, x_{n}\right)$ on $K_{n}$ such that $\max \left|F_{*}^{(n)}\right| \leqslant \max \left|F^{(n)}\right|$ and if $g^{(n)}$ is the distribution function of $F_{*}^{(n)}$, then $g^{(n)} \rightarrow f^{(0)}$ and

$$
\int_{0}^{1} \cdots \int_{0}^{1} F^{(n)}\left(x_{1}, \ldots, x_{n}\right) \cdot F_{*}^{(n)}\left(x_{1}, \ldots, x_{n}\right) d x_{1} \cdots d x_{n} \rightarrow(T F(x))^{2} .
$$

Let us take a bounded continuous functional $F^{*}$ equal to $F_{*}^{(n)}\left(x_{1}, \ldots, x_{n}\right)$ on $X_{n}$ for all even values of $n$ and equal to $F^{(n)}\left(x_{1}, \ldots, x_{n}\right)$ on $X_{n}$ for all odd $n$ (the sets $X_{n}$ are the same as in Theorem 2.1). Obviously the distributions of functions induced by $F^{*}(x)$ on $K_{n}$ converge weakly to $f^{(0)}$ for $n \rightarrow \infty$. Thus there exists a maximal algebra of bounded continuous Gâteaux-Levy integrable functionals containing $F^{*}(x)$. On the other hand, this algebra does not contain $F(x)$, since the functional $F(x) \cdot F^{*}(x)$ is not integrable. We have arrived at a contradiction which proves the theorem. Q.E.D.

Up to now we have considered only Gâteaux-Levy integrability. A similar study can be made for the $\psi$-integral.

EXAMPLE 3.3. A bounded continuous functional $\Omega(x)$, which is not $\psi$ integrable for any $\psi \in \Psi \backslash X$.

Construction. Take the sets $K_{2^{n}} \subset X$ (see Definition 2.1) and a sequence of numbers $1 / 2>p_{1}>p_{2}>\cdots>p_{q}>\cdots, p_{q} \rightarrow 0$. Consider the sets in $K_{2}$ :

$$
\begin{aligned}
X_{n, q}=\left\{\left(x_{1}, \ldots, x_{2^{n}}\right):\left|x_{1}-x_{2}\right|\right. & +\left|x_{3}-x_{4}\right| \\
& \left.+\cdots+\left|x_{2^{n}-1}-x_{2^{n}}\right| \geqslant 2^{n-1} \cdot p_{q}\right\} .
\end{aligned}
$$

For any pair of positive integers $n_{1} \neq n_{2}$ we have $\rho\left(X_{n_{1}, q}, X_{n_{2}, q}\right) \geqslant p_{q} / 2$. Let $U_{n, q}$ be an $\epsilon_{n, q}$-neighborhood of $X_{n, q}$ in $X$, where the numbers $\epsilon_{n, q}$ are selected so that $\bar{U}_{n_{1}, q} \cap \bar{U}_{n_{2}, q}=\varnothing$ if $n_{1} \neq n_{2}$ and $\lim _{n \rightarrow \infty} \epsilon_{n, q}=0$. Consider now for every odd $n$ a continuous functional $\Omega_{n, q} \in G(X)$ equal to $2^{-q}$ on $X_{n, q}$, vanishing 
on $X \backslash U_{n, q}$ and such that $0 \leqslant \Omega_{n, q}(x) \leqslant 2^{-q}$. Set

$$
\Omega_{q}(x)=\sum_{n=1}^{\infty} \Omega_{2 n-1, q}(x) .
$$

Evidently $0 \leqslant \Omega_{q}(x) \leqslant 2^{-q}$. Set

$$
\Omega(x)=\sum_{q=1}^{\infty} \Omega_{q}(x) .
$$

Let $\psi \in \Psi \backslash X$. Let us take a number $p_{q_{0}}$ such that

$$
\int_{0}^{1} \int_{0}^{1} \int_{0}^{1}|\psi(t, x)-\psi(t, y)| d t d x d y>p_{q_{0}} .
$$

It can be easily proved that the functional $\Omega_{q_{0}}(x)$ is not $\psi$-integrable. In fact, if $\Omega_{q_{0}}\left(x_{1}, \ldots, x_{2^{n}}\right)$ are the functions induced by $\Omega_{q_{0}}$ on the cubes $K_{2^{n}}$, then

$$
\begin{gathered}
\lim _{n \rightarrow \infty} \int_{0}^{1} \cdots \int_{0}^{1} \Omega_{q_{0}}\left(\psi_{1}^{\left(2^{2 n-1}\right)}\left(x_{1}\right), \ldots, \psi_{2^{2 n-1}}^{\left(2^{2 n-1}\right)}\left(x_{2^{2 n-1}}\right)\right) d x_{1} \cdots d x_{2^{2 n-1}}=2^{-q_{0}}, \\
\lim _{n \rightarrow \infty} \int_{0}^{1} \cdots \int_{0}^{1} \Omega_{q_{0}}\left(\psi_{1}^{\left(2^{2 n}\right)}\left(x_{1}\right), \ldots, \psi_{2^{2 n}}^{(2 n)}\left(x_{2^{2 n}}\right)\right) d x_{1} \cdots d x_{2^{2 n}}=0
\end{gathered}
$$

(here we use Definition 2.2 of $\psi$-integral).

The functional $\Sigma_{q>q_{0}} \Omega_{q}(x)$ vanishes on the sets $X_{n, q_{0}}$ for even $n$ and hence the functional $\Sigma_{q=q_{0}}^{\infty} \Omega_{q}(x)$ is not $\psi$-integrable. There exists only a finite number of sets $U_{n^{\prime}, q^{\prime}}$, where $n^{\prime}$ is an odd number and $q^{\prime}<q_{0}$, which have a nonempty intersection with the sets $X_{n, q_{0}}$, where $n$ is an even number. According to construction, $\Sigma_{q=1}^{q_{0}-1} \Omega_{q}(x)=\Omega^{(1)}(x)+\Omega^{(2)}(x)$, where $\Omega^{(1)}(x)$ is the sum of functionals $\Omega_{n^{\prime}, q^{\prime}}(x)$ and therefore $\Omega^{(1)}(x) \in G(X)$. Consequently $\Omega^{(1)}(x)$ is $\psi$-integrable. The functional $\Omega^{(2)}(x)$ vanishes on $X_{n, q_{0}}$ for any even $n$ and $\Omega^{(2)}(x) \geqslant 0$. Thus the functional $\Omega(x)$ is not $\psi$-integrable.

\section{Compactifications of the space $X$.}

THEOREM 4.1. A Banach algebra of bounded continuous functionals $A(X)$ determines a compactification of $X$, if and only if for any function $x_{0}(t)$ and for any $\psi(t, x) \in \Psi, x_{0}(t) \neq \psi(t, x)$, there exists a functional $F(x) \in A(X)$ and $a$ functional $F^{\prime}(x)$ of integral form such that $F^{\prime}(x) \leqslant F(x)$ and $F\left(x_{0}(t)\right)<T_{\psi} F^{\prime}(x)$.

Proof. Necessity. Let $F^{\prime}(x)$ be a functional of integral form such that $T_{\psi} F^{\prime}(x)-F^{\prime}\left(x_{0}\right)=d>0$. Consider the following open set in $X: U=\{x(t)$ : $\left.\left|F^{\prime}(x)-F^{\prime}\left(x_{0}\right)\right|<d / 2\right\}$. The functional $F(x) \in A(X) ; F\left(x_{0}\right)=F^{\prime}\left(x_{0}\right)+d / 2$; $F(y)=\sup F^{\prime}(x)$ if $y \in X \backslash U ; F\left(x_{0}\right) \leqslant F(x) \leqslant \sup F^{\prime}(x)$. Obviously $F^{\prime}(x) \leqslant$ $F(x)$ and

$$
F\left(x_{0}\right)=F^{\prime}\left(x_{0}\right)+d / 2<T_{\psi} F^{\prime}(x)=F^{\prime}\left(x_{0}\right)+d
$$


Sufficiency. From the Tychonoff theorem it is sufficient to prove that for any function $x_{0}(t)$ and for any closed set $Y \subset X\left(x_{0}(t) \notin Y\right)$ there exists a functional $F_{0}(x) \in A(X)$ and a number $\epsilon>0$ so that $F_{0}\left(x_{0}(t)\right)<F_{0}(x)-\epsilon$ if $x \in Y$. Let $[Y]$ be the closure of $Y$ in $g X$. For every point $a_{\psi} \in[Y]$ there exists a functional $F_{\psi}(x) \in A(X)$ and a functional integral form $F^{\prime}(x)$ so that $F^{\prime}(x) \leqslant F_{\psi}(x)$ and $0=F_{\psi}\left(x_{0}(t)\right)<T_{\psi} F^{\prime}(x)=F^{\prime}\left(a_{\psi}\right)$. There exists a neighborhood $U^{\prime}\left(a_{\psi}\right)$ of the point $a_{\psi}$ in $g X$ and a number $\epsilon_{\psi}>0$ so that, if $x^{\prime} \in U^{\prime}\left(a_{\psi}\right)$, then $0=F_{\psi}\left(x_{0}(t)\right)<F^{\prime}\left(x^{\prime}\right)-\epsilon_{\psi}$. Let $U_{\psi}=X \cap U^{\prime}\left(a_{\psi}\right)$. If $x \in U_{\psi}$, then $0=$ $F_{\psi}\left(x_{0}(t)\right)<F_{\psi}(x)-\epsilon_{\psi}$. As the set $[Y]$ is compact, a finite number of points $a_{\psi_{1}}, \ldots, a_{\psi_{k}}$ exist, so that $[Y] \subset \bigcup_{i=1}^{k} U^{\prime}\left(a_{\psi_{i}}\right)$. It can be easily seen that $Y \subset \bigcup_{i=1}^{k} U_{\psi_{i}}$ and so for every point $x \in Y$ we have

$$
\begin{aligned}
0 & =\max \left\{F_{\psi_{1}}\left(x_{0}(t)\right), \ldots, F_{\psi_{k}}\left(x_{0}(t)\right)\right\} \\
& <\max \left\{F_{\psi_{1}}(x), \ldots, F_{\psi_{k}}(x)\right\}-\min \left(\epsilon_{\psi_{1}}, \ldots, \epsilon_{\psi_{k}}\right) .
\end{aligned}
$$

For $F_{0}(x)$ we can take the functional $\max \left\{F_{\psi_{1}}(x), \ldots, F_{\psi_{k}}(x)\right\}$ and $\epsilon=$ $\min \left(\epsilon_{\psi_{1}}, \ldots, \epsilon_{\psi_{k}}\right)$. Q.E.D.

Let $F_{n}(x)$ be a sequence of bounded continuous functionals. We say that $F_{n}(x)$ determines a compactification of $X$ if the uniform closure of the algebra, generated by the elements $F_{n}(x)$, is an algebra of bounded continuous functionals determining a compactification of $X$. The following theorem is the corollary of Theorem 4.1.

THEOREM 4.2. A sequence $F_{n}(x)$ of bounded continuous functionals of integral form determines a compactification of the space $X$ if and only if for any function $x_{0}(t)$ and any $\psi(t, x) \in \Psi, x_{0}(t) \neq \psi(t, x)$, there exists a number $n$ such that $F_{n}\left(x_{0}(t)\right) \neq T_{\psi} F_{n}(x)$.

Let $\varphi_{k}(t)$ be a basis of the space $L_{2}(0,1)$. Let $\alpha>0$ be fixed, $\alpha \neq 1$. A direct application of the Tychonoff theorem yields the result that the sequence of functionals of integral form

$$
F_{0}(x)=\int_{0}^{1} x^{\alpha}(t) d t, F_{1}(x)=\int_{0}^{1} \varphi_{1}(t) x(t) d t, \ldots, F_{k}(x)=\int_{0}^{1} \varphi_{k}(t) x(t) d t, \ldots
$$

determines a compactification of the space $X$. Let us prove it with the help of Theorem 4.2. Let $x_{0}(t) \in X, \psi(t, x) \in \Psi, x_{0}(t) \neq \psi(t, x)$. There are two possibilities:

(1) There exists $n$ such that

$$
\begin{aligned}
& F_{n}\left(x_{0}(t)\right)=\int_{0}^{1} \varphi_{n}(t) x_{0}(t) d t \neq \int_{0}^{1} \int_{0}^{1} \varphi_{n}(t) \psi(t, x) d t d x=T_{\psi} F_{n}(x) . \\
& \text { (2) } x_{0}(t)=\int_{0}^{1} \psi(t, x) d x . \text { Then if } \alpha<1
\end{aligned}
$$




$$
F_{0}\left(x_{0}(t)\right)=\int_{0}^{1} x_{0}^{\alpha}(t) d t>\int_{0}^{1} \int_{0}^{1} \psi^{\alpha}(t, x) d t d x=T_{\psi} F_{0}(x)
$$

if $\alpha>1$,

$$
F_{0}\left(x_{0}(t)\right)=\int_{0}^{1} x_{0}^{\alpha}(t) d t<\int_{0}^{1} \int_{0}^{1} \psi^{\alpha}(t, x) d t d x=T_{\psi} F_{0}(x)
$$

The assertions of Theorem 4.2 hold.

5. Limit theorems for functionals on measurable random processes.

THEOREM 5.1. Let $\stackrel{\leftrightarrow}{X}$ be a separable metric space such that:

1. There are probability measures $P_{1}, \ldots, P_{n}, \ldots$ and the probability measure $P$ defined on Borel sets $X$.

2. There exists a Banach algebra $A(X)$ of bounded continuous functions on $\dot{X}$, which determines a compactification $a \dot{X}$ of the space $\dot{X}$ and such that for any $F(x) \in A(\stackrel{X}{)})$ we have

$$
\lim _{n \rightarrow \infty} \int F(x) d P_{n}=\int F(x) d P
$$

Then equality (5.1) holds for every bounded continuous function on the space $\stackrel{X}{X}$, that is, the sequence of probabilities $P_{n}$ converges weakly to the probability $P$.

Proof. Since the space $\dot{X}$ is separable, there exists a separable Banach algebra $A^{\prime}(\stackrel{\circ}{X}) \subset A(\stackrel{X}{X})$, which determines a metric compactification of $\dot{X}$. For simplicity, we consider the algebra $A(X)$ to be separable and $a X^{\circ}$ to be compact metric. Let us extend in a natural way the probabilities $P_{1}, \ldots, P_{n}, \ldots$ and $P$ onto $a \dot{X}$ and denote them on $a \stackrel{\circ}{X}$ by $\mu_{1}, \ldots, \mu_{n}$ and $\mu$. If $V$ is an open set in $a \dot{X}$, then

$$
\mu_{1}(V)=P_{1}(V \cap \stackrel{\circ}{X}), \ldots, \mu_{n}(V)=P_{n}(V \cap \stackrel{\circ}{X}), \ldots, \mu(V)=P(V \cap \stackrel{\circ}{X}) .
$$

By hypothesis for any continuous function $F$ on $a \hat{X}$ we have $\lim _{n \rightarrow \infty} \int F d \mu_{n}=$ $\int F d \mu$, that is, measures $\mu_{n}$ converge weakly to the measure $\mu$ and that is why

$$
\lim _{n \rightarrow \infty} \mu_{n}(V)=\mu(V)
$$

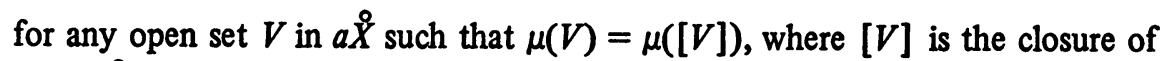

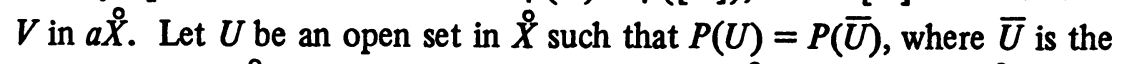
closure of $U$ in $\dot{X}$. There exists an open set $V$ in $a \dot{X}$ such that $U=\dot{X} \cap V$ and $\bar{U}=\stackrel{X}{X} \cap[V]$. Obviously $\mu_{1}(V)=P_{1}(U), \ldots, \mu_{n}(V)=P_{n}(U), \ldots$ and $\mu(V)=$ $\mu([V])=P(U)$. From (5.2) it follows that $\lim _{n \rightarrow \infty} P_{n}(U)=P(U)$. The probability measures $P_{n}$ converge weakly to the probability measure $P$. Q.E.D.

Let us now remember the definition of a measurable random process proposed by Doob. Let $\xi(t)$ be a random process defined on $[0,1]$, i.e. there exists a 
probability space $\Omega=\{\omega\}$ and to every point $t \in[0,1]$ corresponds a random variable $\xi(t, \omega)$ defined on the space $\Omega$. Consider $\xi(t, \omega)$ as a function of two variables defined on the product $[0,1]$ and the space $\Omega$. There exists a measure $\mu$ defined on this product which is the product of the Lebesgue measure on $[0,1]$ and the probability on the space $\Omega$. If the function $\xi(t, \omega)$ is a measurable function with respect to measure $\mu$, the random process $\xi(t)$ is said to be measurable.

Suppose that $\xi(t)$ is a measurable process, $0 \leqslant \xi(t) \leqslant 1$, for all $t \in[0,1]$. Then almost all the sample functions of this process are Lebesgue measurable with range between 0 and 1 . Note that, in general, there may exist two different sample functions of the process $\xi(t)$ which are equal on a set of measure 1 in the interval $[0,1]$. It is important that any continuous functional $F(x)$ on $X$ can be considered as a random variable $F(\xi(t, \omega))$ on $\Omega$.

Definition 5.1. The sequence of random processes $\xi_{n}(t)$ converges to the random process $\xi(t)(0 \leqslant t \leqslant 1)$ if for any positive integer $k$ and for any sequence $n_{i}$ of positive integers there exists a subsequence $n_{i_{j}}$ and a set $A_{k}$ of Lebesgue measure 1 in the $k$-dimensional unit cube $\left(0 \leqslant t_{1} \leqslant 1, \ldots, 0 \leqslant t_{k} \leqslant 1\right)$ such that for any fixed point $\left(t_{1}, \ldots, t_{k}\right) \in A_{k}$ the joint distributions $\Lambda_{j}$ of the random variables $\xi_{n_{i_{j}}}\left(t_{1}\right), \xi_{n_{i_{j}}}\left(t_{2}\right), \ldots, \xi_{n_{i_{j}}}\left(t_{k}\right)$ converge weakly to the joint distribution of the random variables $\xi\left(t_{1}\right), \xi\left(t_{2}\right), \ldots, \xi\left(t_{k}\right)$.

THEOREM 5.2. Let $\xi_{n}(t)$ be a sequence of measurable random processes and $\xi(t)$ be also a measurable random process $\left(0 \leqslant t \leqslant 1,0 \leqslant \xi_{n}(t) \leqslant 1\right.$ for all $n$ and $t, 0 \leqslant \xi(t) \leqslant 1)$. The following assertions are equivalent:

A. The sequence $\xi_{n}(t)$ converges to $\xi(t)$.

B. For any continuous and bounded functional $F(x)$

$$
\lim _{n \rightarrow \infty} E F\left(\xi_{n}(t)\right)=E F(\xi(t)) \text {. }
$$

C. There exists a sequence of functionals of the integral form $F_{m}(x)$ such that:

(a) for any $x_{0}(t) \in X$ and any $\psi(t, x) \in \Psi$, where $x_{0}(t) \neq \psi(t, x)$ there exists a number $m^{\prime}$ such that $F_{m^{\prime}}\left(x_{0}(t)\right) \neq T_{\psi} F_{m^{\prime}}(x)$;

(b) for any $m$ the joint distributions $\Lambda_{n}$ of the random variables $F_{1}\left(\xi_{n}(t)\right), \ldots, F_{m}\left(\xi_{n}(t)\right)$ converge weakly to the joint distribution of the random variables $F_{1}(\xi(t)), \ldots, F_{m}(\xi(t))$.

Proof. We shall prove that $A \Rightarrow B \Rightarrow C \Rightarrow B \Rightarrow A$.

1. $\mathrm{A} \Rightarrow \mathrm{B}$. Set

$$
F(x)=\int_{0}^{1} \cdots \int_{0}^{1} \phi\left(t_{1}, \ldots, t_{k} ; x\left(t_{1}\right), \ldots, x\left(t_{k}\right)\right) d t_{1} \cdots d t_{k}
$$

functional of the integral form. Obviously 


$$
\begin{aligned}
& \lim _{n \rightarrow \infty} E F\left(\xi_{n}(t)\right)=\lim _{n \rightarrow \infty} E\left[\int_{0}^{1} \cdots \int_{0}^{1} \phi\left(t_{1}, \ldots, t_{k} ; \xi_{n}\left(t_{1}\right), \ldots, \xi_{n}\left(t_{k}\right)\right) d t_{1} \cdots d t_{k}\right] \\
& \quad=\lim _{n \rightarrow \infty} \int_{0}^{1} \cdots \int_{0}^{1}\left[E \phi\left(t_{1}, \ldots, t_{k} ; \xi_{n}\left(t_{1}\right), \ldots, \xi_{n}\left(t_{k}\right)\right)\right] d t_{1} \cdots d t_{k} \\
& \quad=\int_{0}^{1} \cdots \int_{0}^{1}\left[E \phi\left(t_{1}, \ldots, t_{k} ; \xi\left(t_{1}\right), \ldots, \xi\left(t_{k}\right)\right)\right] d t_{1} \cdots d t_{k} \\
& =E\left[\int_{0}^{1} \cdots \int_{0}^{1} \phi\left(t_{1}, \ldots, t_{k} ; \xi\left(t_{1}\right), \ldots, \xi\left(t_{k}\right)\right) d t_{1} \cdots d t_{k}\right]=E F(\xi(t)) .
\end{aligned}
$$

Since $G(X)$ determines the compactification of $X$, it follows, by virtue of Theorem 5.1, that (5.3) is valid for any bounded continuous functional on $X$.

2. $B \Rightarrow C$ is evident.

3. $C \Rightarrow B$. This follows from Theorems 4.2 and 5.1.

4. $B \Rightarrow A$.

Let $K$ be a set of measurable functions defined on the $k$-dimensional unit cube $\left(0 \leqslant t_{1} \leqslant 1, \ldots, 0 \leqslant t_{k} \leqslant 1\right)$. If $x\left(t_{1}, \ldots, t_{k}\right) \in K$, then $0 \leqslant$ $x\left(t_{1}, \ldots, t_{k}\right) \leqslant 1$. The set $K$ is closed with respect to the metric

$$
\rho\left(x_{1}, x_{2}\right)=\int_{0}^{1} \cdots \int_{0}^{1}\left|x_{1}\left(t_{1}, \ldots, t_{k}\right)-x_{2}\left(t_{1}, \ldots, t_{k}\right)\right| d t_{1} \cdots d t_{k}
$$

From the Fréchet-Kolmogoroff theorem (see $[10, X, 1]$ ) it follows that the set $K$ is compact in metric (5.4) if and only if for any $\epsilon>0$ there exists $\delta>0$ such that for any sample of nonnegative numbers $\tau_{1}, \ldots, \tau_{k}\left(\delta \geqslant \max _{1<i<k} \tau_{i}\right)$ and for any function $x\left(t_{1}, \ldots, t_{k}\right) \in K$

$$
\int_{0}^{1} \cdots \int_{0}^{1}\left|x\left(t_{1}, \ldots, t_{k}\right)-x\left(t_{1}+\tau_{1}, \ldots, t_{k}+\tau_{k}\right)\right| d t_{1} \cdots d t_{k}<\epsilon,
$$

where the addition $t_{i}+\tau_{i}$ is considered modulo 1 .

Consider the probabilities $P_{1}, \ldots, P_{n}, \ldots$ and $P$ of the processes $\xi_{1}(t), \ldots$, $\xi_{n}(t), \ldots$ and $\xi(t)$ on $X$. If B holds, then for any $\epsilon>0$ there exists a compact $L \subset X$ such that $P_{n}(L) \geqslant 1-\epsilon$ for all $n$ (see $[6, I X, 1]$ ). Thus by virtue of the Fréchet-Kolmogoroff theorem for the functions of one variable we get

$$
\lim _{n \rightarrow \infty ; \tau \rightarrow 0} E \int_{0}^{1}\left|\xi_{n}(t)-\xi_{n}(t+\tau)\right| d t=0
$$

where the addition $t+\tau$ is considered modulo 1 . Consider a sequence $n_{i}$ and the sequence of measurable functions on the $k$-dimensional unit cube:

$$
f_{i}\left(t_{1}, \ldots, t_{k}\right)=E \xi_{n_{i}}^{p_{1}}\left(t_{1}\right) \times \cdots \times \xi_{n_{i}}^{p_{k}}\left(t_{k}\right)
$$

$p_{1}, \ldots, p_{k}$-sample of nonnegative numbers. Since 


$$
\begin{aligned}
& \int_{0}^{1} \cdots \int_{0}^{1}\left|f_{i}\left(t_{1}, \ldots, t_{k}\right)-f_{i}\left(t_{1}+\tau_{1}, \ldots, t_{k}+\tau_{k}\right)\right| d t_{1} \cdots d t_{k} \\
& \leqslant \int_{0}^{1} \cdots \int_{0}^{1}\left\{E \mid \xi_{n_{i}}^{p_{1}}\left(t_{1}\right) \times \cdots \times \xi_{n_{i}}^{p_{k}}\left(t_{k}\right)\right. \\
& \left.\quad-\xi_{n_{i}}^{p_{1}}\left(t_{1}+\tau_{1}\right) \times \cdots \times \xi_{n_{i}}^{p_{k}}\left(t_{k}+\tau_{k}\right) \mid\right\} d t_{1} \cdots d t_{k},
\end{aligned}
$$

it follows, by virtue of (5.5) and the Fréchet-Kolmogoroff theorem, that the closure of the sequence $f_{i}\left(t_{1}, \ldots, t_{k}\right)$ is compact. Thus there exists a subsequence $i_{j}$ and a measurable function $f\left(t_{1}, \ldots, t_{k}\right)$ such that $f_{i_{j}}\left(t_{1}, \ldots, t_{k}\right)$ converges almost everywhere to $f\left(t_{1}, \ldots, t_{k}\right)$. In fact, we have $f\left(t_{1}, \ldots, t_{k}\right)=$ $E \xi^{p}\left(t_{1}\right) \times \cdots \times \xi^{p_{k}}\left(t_{k}\right)$ on a set of measure 1 in the $k$-dimensional cube. Suppose this is not true. Then there exists a set $D$ of positive Lebesgue measure in the $k$-dimensional unit cube such that say $f\left(t_{1}, \ldots, t_{k}\right)-E \xi^{p}{ }^{1}\left(t_{1}\right) \times \cdots \times$ $\xi^{p} k\left(t_{k}\right) \geqslant \epsilon>0$ for all points $\left(t_{1}, \ldots, t_{k}\right) \in D$. Hence

$$
\begin{aligned}
& \lim _{j \rightarrow \infty} E \int \underset{D}{\ldots \int} \xi_{n_{i_{j}}}^{p_{1}}\left(t_{1}\right) \times \cdots \times \xi_{n_{i_{j}}}^{p_{1}}\left(t_{k}\right) d t_{1} \cdots d t_{k} \\
& \geqslant \mu(D) \cdot \epsilon+E \int \underset{D}{\int} \int \xi^{p_{1}}\left(t_{1}\right) \times \cdots \times \xi^{p_{k}}\left(t_{k}\right) d t_{1} \cdots d t_{k} .
\end{aligned}
$$

But this inequality contradicts assertion $B$, since the functional

$$
F(x)=\int \underset{D}{\cdots} \int^{p_{1}}\left(t_{1}\right) \times \cdots \times x^{p_{k}}\left(t_{k}\right) d t_{1} \cdots d t_{k}
$$

is obviously bounded and continuous. Therefore A obviously holds. Q.E.D.

REMARK. We say that finite-dimensional distributions of the random processes $\xi_{n}(t)$ converge to the finite-dimensional distributions of the random process $\xi(t)$ if for any finite sample of points $\left(t_{1}, \ldots, t_{k}\right)$ the joint distributions $\Lambda_{n}$ of the random variables $\xi_{n}\left(t_{1}\right), \ldots, \xi_{n}\left(t_{k}\right)$ converge weakly to the joint distribution of the random variables $\xi\left(t_{1}\right), \ldots, \xi\left(t_{k}\right)$. If $\xi_{n}(t)$ and $\xi(t) \quad\left(0 \leqslant t \leqslant 1,0 \leqslant \xi_{n}(t)\right.$ $\leqslant 1,0 \leqslant \xi(t) \leqslant 1)$ are measurable random processes and if the finite-dimensional distributions of $\xi_{n}(t)$ converge to the finite-dimensional distributions of $\xi(t)$, then condition B of Theorem 5.2 holds. If $\xi_{n}(t)$ and $\xi(t) \quad\left(0 \leqslant t \leqslant 1,0 \leqslant \xi_{n}(t) \leqslant 1\right.$, $0 \leqslant \xi(t) \leqslant 1$ ) are either continuous random processes or processes with the sample functions without discontinuities of the second kind, and if their finite-dimensional distributions converge, then (5.3) does not hold, in general, for all bounded continuous functionals (the functionals are considered on the space of continuous functions with a uniform metric, if the processes are continuous, and on the space of functions without discontinuities of the second kind with a Skorokhod metric (see $[6, \mathrm{IX}, 5]$ ), if the processes with the sample functions without discontinuities of the second kind). 
6. Compactifications of spaces of functions. In previous sections we have considered a compactification method for the space $X$ of all functions $x(t) \in$ $L_{2}(0,1)$ such that $0 \leqslant x(t) \leqslant 1$. Some modifications of this method enable us to construct the compactifications for some spaces of functions.

Let $K$ be metric compact with the countably additive nonnegative measure $\nu$ defined on the Borel sets such that $\nu(K)=1$. We suppose, for simplicity, that $v(t)=0$ for all $t \in K$. Let $L_{p}(K)$ be the space of all $\nu$-measurable functions $x(t)$ on $K$ such that $\int_{K}|x(t)|^{p} d v<\infty, p \geqslant 1$. The metric in $L_{p}(K)$ is given by

$$
\rho\left(x_{1}, x_{2}\right)=\left(\int_{k}\left|x_{1}(t)-x_{2}(t)\right|^{p} d \nu\right)^{1 / p} .
$$

I. Compactification of the space $X\left[f_{1}, f_{2}\right]$. Let $f_{1}(t)$ and $f_{2}(t)$ be continuous functions on $K, f_{1}(t) \leqslant f_{2}(t)$. The set $X\left[f_{1}, f_{2}\right]$ consists of all functions $x(t) \in L_{p}(K)$ such that $f_{1}(t) \leqslant x(t) \leqslant f_{2}(t)$. Let $K \cdot R$ be the product of $K$ with the axis $R$ of real numbers. The points on $R$ we denote by $z$. The set $Z \subset$ $K \cdot R$ consists of the points $(t, z)$ such that $f_{1}(t) \leqslant z \leqslant f_{2}(t)$. Let the set $K_{i}$ be isomorphic to $K$ and $K^{\prime}=\Pi_{i=1}^{k} K_{i}$. Let the set $Z_{i}$ be isomorphic to $Z$ (we denote the points of $Z_{i}$ by $\left(t_{i}, z_{i}\right)$ ). The measure $v_{i}$ is defined on $K_{i}$ and is isomorphic to $v$ on $K$. Consider the functionals of integral form

$$
F(x)=\int_{K^{\prime}} \phi\left(t_{1}, \ldots, t_{k}, x\left(t_{1}\right), \ldots, x\left(t_{k}\right)\right) d v_{1} \cdots d v_{k}
$$

on the space $X\left[f_{1}, f_{2}\right]$, where $\phi\left(t_{1}, \ldots, t_{k} ; z_{1}, \ldots, z_{k}\right)$ is the continuous function on the product of the spaces $\Pi_{i=1}^{k} Z_{i}$. We integrate with respect to the product of measures $\nu_{i}$.

Let $\Psi\left[f_{1}, f_{2}\right]$ be the set of all functions $\psi(t, s)$ defined on the product of $K$ with the unit interval $0 \leqslant s \leqslant 1$ and measurable with respect to the product of $\nu$ and the Lebesgue measure on the unit interval and such that $f_{1}(t) \leqslant \psi(t, s)$ $\leqslant f_{2}(t)$.

THEOREM 6.1. The space $X\left[f_{1}, f_{2}\right]$ has a compactification $M^{*}\left[f_{1}, f_{2}\right]$; each point of $M^{*}\left[f_{1}, f_{2}\right]$ can be written as $\mu_{\psi}, \psi \in \Psi\left[f_{1}, f_{2}\right]$. To each $\psi \in$ $\Psi\left[f_{1}, f_{2}\right]$ corresponds $\mu_{\psi} \in M^{*}\left[f_{1}, f_{2}\right]$. The functionals of integral form (6.1) form an algebra and the uniform closure of this algebra is the Banach algebra of all bounded continuous functionals on $X\left[f_{1}, f_{2}\right]$ which have continuous extensions on $M^{*}\left[f_{1}, f_{2}\right]$. The value of the functional (6.1) at a point $\mu_{\psi}$ is $F\left(\mu_{\psi}\right)=\int_{K^{\prime}} \int_{0}^{1} \cdots \int_{0}^{1} \phi\left(t_{1}, \ldots, t_{k} ; \psi\left(t_{1}, s_{1}\right), \ldots, \psi\left(t_{k}, s_{k}\right)\right) d v_{1} \cdots d v_{k} d s_{1} \cdots d s_{k}$.

II. Compactification of the unit sphere in $L_{p}(K)$. Let $S$ be the unit sphere in $L_{p}(K): x(t) \in S$ if $\int_{K}|x(t)|^{p} d \nu=1$. We use the same notations. Let $Z_{0}=K \cdot R$ 
be the product of $K$ and of the axis $R$ of real numbers. Let $M(S)$ be the set of all nonnegative countably additive measures $\mu$ defined on the Borel sets of $Z_{0}$ such that $\mu\left(Z_{0}\right)=1$ and $\int_{Z_{0}}|z|^{p} d \mu \leqslant 1$. The sequence of measures $\mu_{n}$ converges weakly to the measure $\mu$, if for any bounded continuous function $f(t, z)$, defined on $Z_{0}$, we have

$$
\lim _{n \rightarrow \infty} \int_{z_{0}} f(t, z) d \mu_{n}=\int_{z_{0}} f(t, z) d \mu
$$

This convergence makes $M(S)$ a compact metric space. To each function $x(t) \in S$ corresponds the measure $\mu_{x} \in M(S)$. If $A$ is a Borel set of $Z_{0}$, then

$$
\mu_{x}(A)=\int_{K} \chi_{A}(t, x(t)) d v, \quad \text { where } \chi_{A}(t, z)= \begin{cases}1 & \text { if }(t, z) \in A, \\ 0 & \text { if }(t, z) \notin A .\end{cases}
$$

Obviously $\int_{K}|x(t)|^{p} d \nu=\int_{K}|z|^{p} d \mu_{x}=1$. The mapping $x(t) \rightarrow \mu_{x}$ defines a homeomorphism of the space $S$ onto the space of measures $\mu_{x}$. The closure in $M(S)$ of the set of all measures $\mu_{x}$ we denote by $M^{*}(S)$. Obviously $M^{*}(S)$ is a metric compactification of $S$. Let $\mu^{*} \in M(S)$. The measure $\mu^{*} \in M^{*}(S)$ if and only if for any Borel set $B \subset K \mu^{*}\left(B^{\prime}\right)=\nu(B)\left(B^{\prime}=\{(t, z): t \in B\}\right)$.

Let $\Psi(S)$ be the set of functions $\psi(t, s)$ defined on the product of $K$ and the unit interval $0 \leqslant s \leqslant 1$ and measurable with respect to the product of the measure $\nu$ and of the Lebesgue measure on the unit interval and

$$
\int_{K} \int_{0}^{1}|\psi(t, s)| p d v d s \leqslant 1
$$

i.e., $\Psi(S)$ is the unit ball in the space $L_{p}(K \times[0,1])$. Consider the measure $\mu_{\psi}$ on $Z_{0}$ :

$$
\mu_{\psi}(A)=\int_{K} \int_{0}^{1} \chi_{A}(t, \psi(t, s)) d \nu d s,
$$

where $A$ is a Borel set in $Z_{0}$. The measure $\mu_{\psi} \in M^{*}(S)$. On the other hand, if $\mu^{*} \in M^{*}(S)$, then there exists $\psi \in \Psi(S)$ such that $\mu^{*} \equiv \mu_{\psi}$. The method of the proof is the same as in the first section: for each real number $z^{\prime}$ there is the measure $\mu_{z^{\prime}}$ on $K$. If $B$ is a Borel set in $K$, then $\mu_{z^{\prime}}(B)=\mu^{*}\{(t, z): t \in B$, $z \leqslant z^{\prime}$ \}. Consider the functionals of integral form

$$
F(x)=\int_{K^{\prime}} \phi\left(t_{1}, \ldots, t_{k} ; x\left(t_{1}\right), \ldots, x\left(t_{k}\right)\right) d v_{1} \cdots d v_{k},
$$

where $\phi\left(t_{1}, \ldots, t_{k} ; z_{1}, \ldots, z_{k}\right)$ is a bounded continuous function on $\Pi_{i=1}^{k} Z_{0}^{(i)}$, $Z_{0}^{(i)}$ isomorphic to $Z_{0}, K^{\prime}=\Pi_{i=1}^{k} K_{i}$, etc.

THEOREM 6.2. The space $S$ has a metric compactification $M^{*}(S)$. Each point of $M^{*}(S)$ can be written as $\mu_{\psi}, \psi \in \Psi(S)$. To each $\psi \in \Psi(S)$ corresponds 
a point $\mu_{\psi} \in M^{*}(S)$. The functionals of integral form (6.2) form the algebra and the uniform closure of this algebra is the Banach algebra of all bounded continuous functionals on $S$ continuously extendable on $M^{*}(S)$. The value of functional (6.2) at a point $\mu_{\psi}$ is

$$
F\left(\mu_{\psi}\right)=\int_{K^{\prime}} \int_{0}^{1} \cdots \int_{0}^{1} \phi\left(t_{1}, \ldots, t_{k} ; \psi\left(t_{1}, s_{1}\right), \ldots, \psi\left(t_{k}, s_{k}\right)\right) d v_{1} \cdots d v_{k} d s_{1} \cdots d s_{k} .
$$

REMARK. The foregoing compactification principle cannot be applied to the case of the unit ball $V$ in $L_{p}(K)$, i.e. to the set of functions $x(t)$ such that $\int_{K}|x(t)|^{p} d \nu \leqslant 1$, since the mapping $x(t) \rightarrow \mu_{x}$ is not a homeomorphism.

III. Compactification of the unit ball $V \subset L_{p}(K)$. The space $Z_{0}$ is a metric locally compact space. There exists a one-point compactification $q Z_{0}$, where $q Z_{0}$ $=Z_{0} \cup\{\infty\}$ (P. S. Alexandroff, see $[7, I, 9,8]$ ).

Let $M\left(q Z_{0}\right)$ be the set of all nonnegative countably additive measures $\mu$ defined on the Borel sets of $q Z_{0}$ such that $\mu\left(q Z_{0}\right) \leqslant 1$. The sequence of measures $\mu_{n}$ converges weakly to the measure $\mu$, if for any continuous function $f(w)$ on $q Z_{0}$ we have

$$
\lim _{n \rightarrow \infty} \int_{q z_{0}} f(w) d \mu_{n}=\int_{q z_{0}} f(w) d \mu .
$$

This convergence makes $M\left(q Z_{0}\right)$ compact metric.

To each $x(t) \in V$ corresponds the measure $\mu_{x}^{\prime} \in M\left(q Z_{0}\right)$ defined by

$$
\mu_{x}^{\prime}(A)=\int_{K} \hat{\chi}_{A}(t, x(t)) d \nu,
$$

where $A$ is a Borel set in $Z_{0}, \hat{\chi}_{A}(t, z)=|z|^{p}$ if $(t, z) \in A$, and $\hat{\chi}_{A}(t, z)=0$ if $(t, z) \notin A$. Obviously $\mu_{x}^{\prime}(\infty)=0$. The mapping $x(t) \rightarrow \mu_{x}^{\prime}$ defines a homeomorphism of $V$ onto the space of measures $\mu_{x}^{\prime}$. The closure in $M\left(q Z_{0}\right)$ of the set of all measures $\mu_{x}^{\prime}$ we denote by $\tilde{M}(V)$. Evidently $\tilde{M}(V)$ is a metric compactification of $V$.

Let $\tilde{\mu} \in \tilde{M}(V)$. Denote by $\tilde{\mu}_{0}$ the measure induced by $\tilde{\mu}$ on $Z_{0}$. There exists a function $\psi(t, s) \in \Psi(S)$ such that

$$
\tilde{\mu}_{0}(A)=\int_{K} \int_{0}^{1} \hat{x}_{A}(t, \psi(t, s)) d v d s,
$$

where $A$ is a Borel set in $Z_{0}$. Indeed consider the sequence of functions $\left\{x_{k}(t)\right\}$ $\subset V$ such that the sequence of measures $\mu_{x_{k}}^{\prime}$ defined by (6.3) converges weakly to $\tilde{\mu}$. Let $\mu_{x_{k}}$ be a sequence of measures in $M^{*}(S)$ determined for the functions $x_{k}(t) \in \Psi(S)$ as above. The sequence $\mu_{x_{k}}$ converges weakly to the measure $\mu_{\psi} \in M^{*}(S)$. The function $\psi$ satisfies (6.4) for all Borel sets $A \subset Z_{0}$. On the other hand, for any function $\psi(t, s) \in \Psi(S)$ and for any nonnegative number $\alpha$ such that

$$
\int_{K} \int_{0}^{1}|\psi(t, s)|^{p} d v d s+\alpha \leqslant 1
$$


there exists a measure $\widetilde{\mu} \in \widetilde{M}(V)$ such that the measure $\widetilde{\mu}_{0}$ induced by $\widetilde{\mu}$ on $Z_{0}$ is given by $(6.4)$ and $\widetilde{\mu}(\infty)=\alpha$. Consider the functional

$$
F(x)=\int_{K} f(t, x(t))|x(t)|^{p} d \nu,
$$

where $f(t, z)$ is a bounded continuous function on $Z_{0}$, which has a continuous extension on $q Z_{0}$. The functional (6.6) is continuously extendable on $\widetilde{M}(V)$ and

$$
F(\widetilde{\mu})=\int_{q z_{0}} f(w) d \tilde{\mu}=\int_{K} \int_{0}^{1} f(t, \psi(t, s))|\psi(t, s)|^{p} d v d s+\alpha \cdot f(\infty) \text {. }
$$

THEOREM 6.3. The space $V$ has a metric compactification $\widetilde{M}(V)$. Each point of $\widetilde{M}(V)$ is determined by the pair $(\psi, \alpha)$, where $\psi \in \Psi(S)$ and $\alpha$ is a nonnegative number, so that $(6.5)$ holds. To each pair $(\psi, \alpha)$ satisfying inequality (6.5) there corresponds a point $\tilde{\mu} \in \tilde{M}(V)$. The functionals $F(x)$ of form (6.6) generate the algebra, its uniform closure being the Banach algebra of all bounded continuous functionals on $V$ which have continuous extension on $\widetilde{M}(V)$. The value of $F(\widetilde{\mu})$ is given by (6.7).

REMARK. Let $S$ be the unit sphere in $L_{p}(K)$. The closure of $S$ in $\widetilde{M}(V)$ is the set $\bar{M}(S)$ of those points $\widetilde{\mu}$ for which the corresponding pairs $(\psi, \alpha)$ satisfy the equality $\int_{K} \int_{0}^{1}|\psi(t, s)|^{p} d v d s+\alpha=1$. The set $\bar{M}(S)$ is the compactification of $S$. It is easy to see that the compactifications $M^{*}(S)$ and $\bar{M}(S)$ are equivalent.

IV. Compactification of the unit ball in $L_{p}(\hat{K})$. Let $\hat{K}$ be a metric locally compact space with the nonnegative countably-additive measure $h$ defined on the Borel subsets. If $Q \subset \hat{K}$ and $Q$ is compact, then we assume that $h(Q)<\infty$. For all $t \in \hat{K}$ we suppose $h(t)=0$. Consider the unit ball $\hat{V}$ of the space $L_{p}(\hat{K})$, i.e., $x(t) \in \hat{V}$ if $\int_{\hat{K}}|x(t)|^{p} d h \leqslant 1$. By analogy to the foregoing set $Z_{0}=\hat{K} \cdot R$, where $R$ is the axis of real numbers. It is clear that $Z_{0}$ is a metric locally compact space. Consider the metric compactification $q Z_{0}$ of the space $Z_{0}$ such that the set $q Z_{0} \backslash Z_{0}$ consists of only one point which we denote by $\infty$. Consider the space of functions $\Psi(\hat{V})$. The function $\psi(t, s)$, where $t \in \hat{K}$ and $0 \leqslant s \leqslant 1$, belongs to $\Psi(\hat{V})$ if $\psi(t, s)$ is measurable with respect to the product of the measure $h$ and the Lebesgue measure on the unit interval and if $\int_{\widehat{K}} \int_{0}^{1}|\psi(t, s)|^{p} d h d s \leqslant 1$. Consider on $\hat{V}$ the functionals

$$
F(x)=\int_{\hat{K}} f(t, x(t))|x(t)|^{p} d h,
$$

where $f(t, z)$ is a bounded continuous function on $Z_{0}$ which has a continuous extension on $q Z_{0}$.

THEOREM 6.4. The space $\hat{V}$ has metric compactification $\widetilde{M}(\hat{V})$. Each point $\widetilde{\mu} \in \widetilde{M}(\hat{V})$ is determined by the pair $(\psi, \alpha)$, where $\psi \in \Psi(\hat{V}), \alpha \geqslant 0$ and $\int_{\hat{K}} \int_{0}^{1}|\psi(t, s)|^{p} d h d s+\alpha \leqslant 1$. The function $x(t) \in \hat{V}$ is given by the pair $(x(t), 0)$. 
For each pair $(\psi, \alpha)$, where $\psi \in \Psi(\hat{V}), \alpha \geqslant 0$ and $\int_{\hat{K}} \int_{0}^{1}|\psi(t, s)|^{p} d h d s+\alpha \leqslant 1$ there exists the corresponding point $\widetilde{\mu} \in \widetilde{M}(\hat{V})$. The functionals $F(x)$ of form (6.8) generate the algebra, its uniform closure being the Banach algebra of all bounded continuous functionals on $\hat{V}$ continuously extendable on $\widetilde{M}(\hat{V})$ and

$$
F(\widetilde{\mu})=\int_{\hat{K}} \int_{0}^{1} f(t, \psi(t, s)) \mid \psi(t, s) p^{p^{\prime}} d h d s+\alpha \cdot f(\infty) .
$$

\section{BIBLIOGRAPHY}

1. R. Gâteaux, Bull. Soc. Math. France 47 (1919).

2. P. Lévy, Problèmes concrets d'analyse fonctionnelle, 2nd ed., Gauthier-Villars, Paris, 1951. MR 12, 834.

3. E. Borel, Introduction géometrique á quelques théories physiques, Gauthier-Villars, Paris, 1914.

4. P. S. Aleksandrov, Introduction to the general theory of sets and functions, GITTL, Moscow, 1948. (Russian) MR 12, 682.

5. I. M. Gel' fand and A. N. Kolmogoroff, On rings of continuous functions on topological spaces, C. R. (Dokl.) Acad. Sci. USSR 22 (1939), 11-15.

6. I. I. Gihman and A. V. Skorohod, Introduction to the theory of random processes, "Nauka", Moscow, 1965; English transl., Saunders, Philadelphia, Pa., 1969. MR 33 \#6689; 40 \#923.

7. N. Bourbaki, Éléments de mathématique. Part 1. Les structures fondamentales d'analyse. Livre III: Topologie générale. Chap. I, Actualités Sci. Indust., no. 858, Hermann, Paris, 1940. MR 3, 55.

8. M. H. Stone, Applications of the theory of Boolean rings to general topology, Trans. Amer. Math. Soc. 41 (1937), 375-481.

9. I. Gel'fand, D. Raikov and G. E. Silov, Commutative normed rings, Fizmatgiz, Moscow, 1960; English transl., Chelsea, New York, 1964. MR 23 \#A1242; 34 \#4940.

10. K. Yosida, Functional analysis, Die Grundlehren der math. Wissenschaften, Band 123, Academic Press, New York; Springer-Verlag, Berlin, 1965. MR 31 \#5054. ISRAEL

DEPARTMENT OF MATHEMATICS, BAR-ILAN UNIVERSITY, RAMAT-GAN, 